\title{
Relative Perturbation Theory for Quadratic Hermitian Eigenvalue Problem
}

\author{
Peter Benner* $\quad$ Xin Liang $^{\dagger} \quad$ Suzana Miodragović Ninoslav $^{\ddagger}$ Truhar $^{\S}$
}

October 23, 2020

\begin{abstract}
In this paper, we derive new relative perturbation bounds for eigenvectors and eigenvalues for regular quadratic eigenvalue problems of the form $\left(\lambda^{2} M+\lambda C+K\right) x=0$, where $M$ and $K$ are nonsingular Hermitian matrices and $C$ is a general Hermitian matrix. These results are based on new relative perturbation bounds for an equivalent regular Hermitian matrix pair $A-\lambda B$. The new bounds can be applied to quadratic eigenvalue problems appearing in many relevant applications, such as mechanical models with indefinite damping. The quality of our bounds is demonstrated by several numerical experiments.
\end{abstract}

AMS subject classifications. 15A18, 15A42, 65F15, 65F35, 65G99

Keywords. relative perturbation theory, quadratic eigenvalue problem, Hermitian quadratic matrix polynomial, Hermitian matrix pair

\section{Introduction}

The quadratic eigenvalue problem (QEP) is to find scalars $\lambda$ and nonzero vectors $x$ satisfying

$$
\left(\lambda^{2} M+\lambda C+K\right) x=0,
$$

where $M, C$ and $K$ are $n \times n$ complex matrices. The solution to the QEP is required in many applications arising in the dynamic analysis of structural mechanical and acoustic systems, in electronic circuit simulation, in fluid mechanics, in modeling micro-electronic-mechanical systems (MEMS), and so on. The number of applications of the QEP is constantly growing. In [11, 19], the theoretical background of the QEP and other polynomial eigenvalue problems is extensively

\footnotetext{
${ }^{*}$ Max Planck Institute for Dynamics of Complex Technical Systems, Sandtorstraße 1, 39106 Magdeburg, Germany. E-mail: benner@mpi-magdeburg.mpg.de.

${ }^{\dagger}$ Max Planck Institute for Dynamics of Complex Technical Systems, Sandtorstraße 1, 39106 Magdeburg, Germany. E-mail: liangxinslm@tsinghua.edu.cn.

${ }^{\ddagger}$ Department of Mathematics, University of Osijek, Trg Ljudevita Gala 6, 31000 Osijek, Croatia. E-mail: ssusic@mathos.hr.

${ }^{\S}$ Department of Mathematics, University of Osijek, Trg Ljudevita Gala 6, 31000 Osijek, Croatia. E-mail: ntruhar@mathos.hr.
} 
studied; in [28] a survey of applications, mathematical properties, and a variety of numerical solution techniques for the QEP is given.

This paper aims to build relative perturbation bounds for eigenvalues and eigenspaces of the QEP (1.1), where $M, C$, and $K$ are allowed to be Hermitian indefinite matrices, and $C$ can be even singular.

In general, the perturbation theory of matrix or operator eigenvalue problems can be divided into two major branches. One belongs to the so-called standard or absolute perturbation theory which can be found in many well-known textbooks, e.g., [17, 10, 3, 5, 25, 27]. The other, the so-called relative perturbation theory, has became a very active research area since the late 1980s. Some important results of the relative perturbation theory can be found in [8, 2, 9]. The development of such a kind of theory goes back to as early as Kahan's technical report [16] in 1966.

Regarding the perturbation theory for the QEP in a general setting, standard or absolute perturbation bounds are given, for example, in [24, 30, 28], but to the authors' knowledge there are no relative perturbation bounds for eigenvalues and especially for eigenspaces of QEPs where all three coefficient matrices are Hermitian without any further assumptions.

Some results on relative perturbations of the QEP can be found in [29]. There one can find bounds for eigenvectors and eigenvalues of the QEP (1.1), where the matrices $M, C$ and $K$ are positive definite Hermitian matrices and the condition

$$
\left(x^{H} C x\right)^{2}-4\left(x^{H} M x\right)\left(x^{H} K x\right)>0, \quad \forall x \in \mathbb{C}^{n}, x \neq 0
$$

is satisfied, which means that the corresponding QEP is hyperbolic. The hyperbolic QEP is investigated, e.g., in [28, 24].

In this paper we will derive similar bounds for the more general case, that is, the QEP will be regular and $C$ can be any Hermitian matrix.

Such QEPs are interesting because many of the instabilities in mechanical systems are caused by a negative definite or indefinite damping matrix $C$, see for example $[26,1,15,31,18,6]$. Therefore, we will illustrate our results using numerical experiments motivated by these problems.

Perturbation bounds for the regular QEP will be derived by using linearization and perturbation bounds for any regular matrix pair $A-\lambda B$, where $A$ and $B$ are Hermitian matrices, given in Section 3.

There is a vast amount of material in perturbation theory (covering absolute and relative perturbation results) for definite matrix pairs. Here, we will list some of those results related to eigenspace perturbations, where the distance between two eigensubspaces is measured by some trigonometric function of the angle operator $\Theta$ associated with the eigensubspaces $\mathcal{X}=\operatorname{span}(X)$ and $\mathcal{Y}=\operatorname{span}(Y)$ of the unperturbed and perturbed matrix pairs. The angle operator $\Theta$ is defined by

$$
\Theta(\mathcal{X}, \mathcal{Y})=\arcsin \left(P_{\mathcal{X}}-P_{\mathcal{Y}}\right)
$$

where $P_{\mathcal{X}}$ and $P_{\mathcal{Y}}$ are orthogonal projections onto the subspaces $\mathcal{X}$ and $\mathcal{Y}$, of the same dimension (see, e.g., [27]). The eigenvalues of the matrix $\Theta$ represent canonical angles. One can find results for standard perturbation theory and relative perturbation bounds for eigenspaces in $[27,7]$ and in $[14,13,29]$, respectively. For the case when $B$ is Hermitian positive definite, in [14, 13], the authors define the angle operator $\Theta$ in the matrix-dependent scalar product $\langle x, y\rangle_{B}=y^{T} B x$, 
for $x, y \in \mathbb{R}^{n}$. A similar approach is not possible for the case when $B$ is Hermitian indefinite nonsingular. For that case, as it has been shown in [29], it is possible to measure the distance between two subspaces, using the fact that $\|\sin \Theta(\mathcal{X}, \mathcal{Y})\| \rightarrow 0$ if and only if $\left\|Y^{H} B X\right\| \rightarrow 0$. The same idea will be used in this paper.

This paper is organized as follows. In Section 3 we derive our relative perturbation $\sin \Theta$ type theorems and a relative bound on the eigenvalues of regular matrix pairs. We apply these bounds to the QEP and derive relative perturbation bounds for eigenvectors and eigenvalues in Section 4. Numerical examples are given in Section 5 to illustrate our bounds. Finally, some conclusions are summarized in Section 6.

Notation. Throughout this paper, we use $\|\cdot\|_{2},\|\cdot\|_{F}$ and $\|\cdot\|_{\text {ui }}$ to denote the spectral matrix norm, the Frobenius norm and any unitarily invariant matrix norm, respectively. We use $\kappa_{2}(\cdot)$ to denote the spectral condition number. $I_{n}$ is the identity matrix of size $n$, and sometimes the subscript is omitted when the size is clear from the context. If $A$ is a Hermitian positive (semi-)definite matrix, we will write $A \succ 0(A \succeq 0)$, and similarly $A \prec 0(A \preceq 0)$ for a Hermitian negative (semi-)definite $A . A^{+}$is the Moore-Penrose pseudo-inverse of $A$. We use the standard MATLAB ${ }^{\circledR}$ notation $A(:, i)$ for the $i$-th column of the matrix $A$. Also, we denote by $F_{m}$ and $G_{m}$, respectively, the $m \times m$ matrices given by

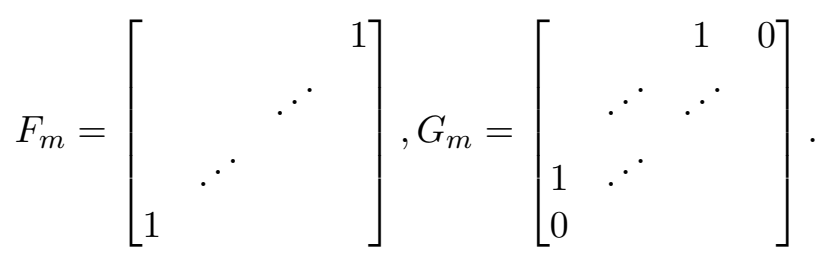

Then the Jordan block of size $m$ corresponding to the eigenvalue $\lambda$ is

$$
J_{m}(\lambda):=\lambda I_{m}+G_{m} F_{m}=\left[\begin{array}{cccc}
\lambda & 1 & & \\
& \ddots & \ddots & \\
& & \ddots & 1 \\
& & & \lambda
\end{array}\right] .
$$

A matrix pair $A-\lambda B$ is sometimes denoted by $(A, B)$. If $A_{1}, B_{1}$ have the same size and so do $A_{2}, B_{2}$, then the matrix pair $\left[\begin{array}{ll}A_{1} & \\ & A_{2}\end{array}\right]-\lambda\left[\begin{array}{ll}B_{1} & \\ & B_{2}\end{array}\right]$ is also written as $\left(A_{1}-\lambda B_{1}\right) \oplus\left(A_{2}-\lambda B_{2}\right)$ or $\left(A_{1}, B_{1}\right) \oplus\left(A_{2}, B_{2}\right)$. For any matrices $W, V$ of appropriate sizes, $W^{H}(A, B) V$ is used to denote the pair $\left(W^{H} A V, W^{H} B V\right)$.

\section{Canonical forms for matrix pairs}

As described in the introduction, we will derive perturbation bounds for regular Hermitian matrix pairs $(A, B)$, and we will use those bounds to derive bounds for the QEP. Thus, in this section, we recall a canonical form of Hermitian matrix pairs in Lemma 2.1, which will play a fundamental role in the discussion of eigenspaces. 
Lemma 2.1 ([12, Theorem 5.10.1], [20, Theorem 6.1]). Every Hermitian matrix pair $(A, B)$ is congruent to a Hermitian matrix pair of the form

$$
\begin{aligned}
& (0,0) \oplus \bigoplus_{j=1}^{p}\left(G_{2 \varepsilon_{j}+1},\left[\begin{array}{lll} 
& & F_{\varepsilon_{j}} \\
F_{\varepsilon_{j}} & &
\end{array}\right]\right) \\
& \oplus \bigoplus_{j=1}^{r} \delta_{j}\left(F_{k_{j}}, G_{k_{j}}\right) \oplus \bigoplus_{j=1}^{q} \eta_{j}\left(\alpha_{j} F_{\ell_{j}}+G_{\ell_{j}}, F_{\ell_{j}}\right) \\
& \oplus \bigoplus_{j=1}^{s}\left(\left[\overline{\beta_{j}} F_{m_{j}}+G_{m_{j}} \quad \beta_{j} F_{m_{j}}+G_{m_{j}}\right], F_{2 m_{j}}\right) .
\end{aligned}
$$

Here, $\varepsilon_{1} \leq \cdots \leq \varepsilon_{p}$ and $k_{1} \leq \cdots \leq k_{p}, l_{1} \leq \cdots \leq l_{q}$ and $m_{1} \leq \cdots \leq m_{s}$ are positive integers, $\alpha_{j}$ are real numbers, $\beta_{j}$ are complex non-real numbers, and $\delta_{j}, \eta_{j}$ are signs $(+1$ or -1$)$. The form is uniquely determined by $(A, B)$ up to a combination of permutations of the following types of blocks:

T0. $(0,0)$;

T1. $\left(G_{2 \varepsilon_{j}+1},\left[\begin{array}{ccc} & F_{\varepsilon_{j}} \\ F_{\varepsilon_{j}} & & \end{array}\right]\right), \quad j=1, \ldots, p ;$

T2. $\delta_{j}\left(F_{k_{j}}, G_{k_{j}}\right), \quad j=1, \ldots, r$;

T3. $\eta_{j}\left(\alpha_{j} F_{\ell_{j}}+G_{\ell_{j}}, F_{\ell_{j}}\right), \quad j=1, \ldots, q$;

T4. $\left.\left.\frac{\left[\bar{\beta}_{j}\right.}{\beta_{j}} F_{m_{j}}+G_{m_{j}} \beta_{j} F_{m_{j}}+G_{m_{j}}\right], F_{2 m_{j}}\right), \quad j=1, \ldots, s$, with possible replacement of $\beta_{j}$ by

Specifically, if $(A, B)$ is a regular pair, then its canonical form only contains blocks of type T2, T3, T4.

To indicate the similarity of the asymptotic behavior between these blocks and the corresponding eigenvalue, in the following we apply a congruence transformation:

1. For blocks of type $\mathbf{T} 3$ with $\alpha_{j} \neq 0$, write

$$
T=\operatorname{diag}\left(\left|\alpha_{j}\right|^{\frac{\ell_{j}-1}{2}},\left|\alpha_{j}\right|^{\frac{\ell_{j}-1}{2}-1}, \ldots,\left|\alpha_{j}\right|^{-\frac{\ell_{j}-1}{2}}\right) .
$$

Then $T^{H} F_{\ell_{j}} T=F_{\ell_{j}}$ and $T^{H} G_{\ell_{j}} T=\left|\alpha_{j}\right| G_{\ell_{j}}$, so the new block pair is of type $\eta_{j}\left(\alpha_{j} F_{\ell_{j}}+\right.$ $\left.\left|\alpha_{j}\right| G_{\ell_{j}}, F_{\ell_{j}}\right)$.

2. For blocks of type $\mathbf{T} \mathbf{4}$, write

$$
T=\operatorname{diag}\left(\left|\beta_{j}\right|^{\frac{m_{j}-1}{2}},\left|\beta_{j}\right|^{\frac{m_{j}-1}{2}-1}, \ldots,\left|\beta_{j}\right|^{-\frac{m_{j}-1}{2}}\right) .
$$


Then $T^{H} F_{m_{j}} T=F_{m_{j}}$ and $T^{H} G_{m_{j}} T=\left|\beta_{j}\right| G_{m_{j}}$. Since

$$
\left[\begin{array}{ll}
T & \\
& T
\end{array}\right]^{H}\left[\begin{array}{ll}
S^{H} & S
\end{array}\right]\left[\begin{array}{ll}
T & \\
& T
\end{array}\right]=\left[\begin{array}{ll}
T^{H} S^{H} T & T^{H} S T
\end{array}\right],
$$

the new block pair is of type

$$
\left(\left[\overline{\beta_{j}} F_{m_{j}}+\left|\overline{\beta_{j}}\right| G_{m_{j}} \beta_{j} F_{m_{j}}+\left|\beta_{j}\right| G_{m_{j}}\right], F_{2 m_{j}}\right) .
$$

From this, we obtain the following variant of the canonical form:

Lemma 2.2. Every regular Hermitian matrix pair $(A, B)$ is congruent to a Hermitian matrix pair of the form

$$
\begin{aligned}
\bigoplus_{j=1}^{r} \delta_{j}\left(F_{k_{j}}, G_{k_{j}}\right) \oplus \bigoplus_{j=1}^{q^{\prime}} \eta_{j}^{\prime}\left(G_{\ell_{j}^{\prime}}, F_{\ell_{j}^{\prime}}\right) & \oplus \bigoplus_{j=1}^{q} \eta_{j}\left(\alpha_{j} F_{\ell_{j}}+\left|\alpha_{j}\right| G_{\ell_{j}}, F_{\ell_{j}}\right) \\
& \oplus \bigoplus_{j=1}^{s}\left(\left[\overline{\beta_{j}} F_{m_{j}}+\left|\overline{\beta_{j}}\right| G_{m_{j}} \quad \beta_{j} F_{m_{j}}+\left|\beta_{j}\right| G_{m_{j}}\right], F_{2 m_{j}}\right) .
\end{aligned}
$$

Here $, k_{j}, l_{j}$ and $m_{j}$ are positive integers, $\alpha_{j}$ are nonzero real numbers, $\beta_{j}$ are complex non-real numbers, and the $\delta_{j}, \eta_{j}^{\prime}, \eta_{j}$ are signs $(+1$ or -1$)$. This form is uniquely determined by $(A, B)$ up to a combination of permutations of the following types of blocks:

R1. $\delta_{j}\left(F_{k_{j}}, G_{k_{j}}\right), \quad j=1, \ldots, r$;

R2. $\eta_{j}^{\prime}\left(G_{\ell_{j}^{\prime}}, F_{\ell_{j}^{\prime}}\right), \quad j=1, \ldots, q^{\prime}$;

R3. $\eta_{j}\left(\alpha_{j} F_{\ell_{j}}+\left|\alpha_{j}\right| G_{\ell_{j}}, F_{\ell_{j}}\right), \quad j=1, \ldots, q$;

R4. $\left(\left[\overline{\beta_{j}} F_{m_{j}}+\left|\overline{\beta_{j}}\right| G_{m_{j}} \beta_{j} F_{m_{j}}+\left|\beta_{j}\right| G_{m_{j}}\right], F_{2 m_{j}}\right), \quad j=1, \ldots, s$, with possible replacement of $\beta_{j}$ by $\overline{\beta_{j}}$.

\section{Relative perturbation bound for a Hermitian matrix pair}

In this section, first we will see that the bound on the angle between the eigenspaces of a regular Hermitian matrix pair is related to a bound on the solutions to structured Sylvester equations $S X-X S^{\prime}=T$ with coefficients $S, S^{\prime}, T$ of special structure. Our approach more or less follows the lines of Davis and Kahan [7] and is very closely related to the work of $\mathrm{Li}$ [22, 23]. 


\subsection{Bound on the solution of the structured Sylvester equation}

Besides the references given above, structured Sylvester equations and their connection to the $\sin \Theta$-type theorems can be found in, e.g., [21]. According to our needs, we derive Lemma 3.1.

Lemma 3.1. Given two matrices $M, N$ of the same size. Suppose $(\Lambda, \Omega)$ and $\left(\Lambda^{\prime}, \Omega^{\prime}\right)$ are block pairs of the type $\boldsymbol{R} 2, \boldsymbol{R} 3, \boldsymbol{R} 4$ in $(2.2)$. Write $\lambda(\Lambda, \Omega)=\{\underbrace{\lambda, \ldots, \lambda}_{n}\}$ or $\{\underbrace{\lambda, \bar{\lambda}, \ldots, \lambda, \bar{\lambda}}_{2 n}\}$ and $\lambda\left(\Lambda^{\prime}, \Omega^{\prime}\right)=\{\underbrace{\lambda^{\prime}, \ldots, \lambda^{\prime}}_{n^{\prime}}\}$ or $\{\underbrace{\lambda^{\prime}, \overline{\lambda^{\prime}}, \ldots, \lambda^{\prime}, \overline{\lambda^{\prime}}}_{2 n^{\prime}}\}$. For any $\alpha>0, m \in \mathbb{N}$, define

$$
\varphi_{-}(\alpha, m):=\alpha^{1-m} \frac{1-\alpha^{m}}{1-\alpha}, \quad \varphi_{+}(\alpha, m):=\alpha^{-m} \frac{1+\alpha-2 \alpha^{m}}{1-\alpha} .
$$

If $\lambda(\Lambda, \Omega) \cap \lambda\left(\Lambda^{\prime}, \Omega^{\prime}\right)=\emptyset$, then each of the equations

$$
\begin{aligned}
\left(\Omega^{\prime} \Lambda^{\prime}\right)^{H} Y-Y \Omega \Lambda & =-\left(\Omega^{\prime} \Lambda^{\prime}\right)^{H} M+N \Omega \Lambda, \\
Y-\left(F_{n^{\prime}} G_{n^{\prime}}\right)^{H} Y \Omega \Lambda & =-M+\left(F_{n^{\prime}} G_{n^{\prime}}\right)^{H} N \Omega \Lambda,
\end{aligned}
$$

has a unique solution $Y$ whose size is the same as that of $M, N$. For (3.1b) we define $\lambda^{\prime}=\infty$. Then, for both (3.1a) and (3.1b), the solution $Y$ is bounded by

$$
\|Y\|_{F} \leq \phi_{1}\left(\lambda, \lambda^{\prime}, n, n^{\prime}\right)\|M\|_{F}+\phi_{2}\left(\lambda, \lambda^{\prime}, n, n^{\prime}\right)\|N\|_{F},
$$

where

$$
\begin{aligned}
\phi_{1}\left(\lambda, \lambda^{\prime}, n, n^{\prime}\right) & =\left(\begin{array}{c}
n^{\prime}+n-1 \\
n
\end{array}\right) \varphi_{-}(\gamma, n) \varphi_{+}\left(\gamma^{\prime}, n^{\prime}\right), \\
\phi_{2}\left(\lambda, \lambda^{\prime}, n, n^{\prime}\right) & =\left(\begin{array}{c}
n^{\prime}+n-1 \\
n^{\prime}
\end{array}\right) \varphi_{-}\left(\gamma^{\prime}, n^{\prime}\right) \varphi_{+}(\gamma, n), \\
\phi_{1}\left(0, \lambda^{\prime}, n, n^{\prime}\right) & =\left(\begin{array}{c}
n^{\prime}+n-1 \\
n
\end{array}\right)\left|\lambda^{\prime}\right|^{-1} \varphi_{-}\left(\left|\lambda^{\prime}\right|, n-1\right)+1, \\
\phi_{2}\left(0, \lambda^{\prime}, n, n^{\prime}\right) & =\left(\begin{array}{c}
n^{\prime}+n-1 \\
n
\end{array}\right)\left|\lambda^{\prime}\right|^{-1} \varphi_{-}\left(\left|\lambda^{\prime}\right|, n-1\right), \\
\phi_{1}\left(\lambda, 0, n, n^{\prime}\right) & =\left(\begin{array}{c}
n^{\prime}+n-1 \\
n^{\prime}
\end{array}\right)|\lambda|^{-1} \varphi_{-}\left(|\lambda|, n^{\prime}-1\right), \\
\phi_{2}\left(\lambda, 0, n, n^{\prime}\right) & =\left(\begin{array}{c}
n^{\prime}+n-1 \\
n^{\prime}
\end{array}\right)|\lambda|^{-1} \varphi_{-}\left(|\lambda|, n^{\prime}-1\right)+1, \\
\phi_{1}\left(\lambda, \infty, n, n^{\prime}\right) & =2|\lambda| \varphi_{-}\left(\frac{1}{2|\lambda|}, n^{\prime}-1\right)+1, \\
\phi_{2}\left(\lambda, \infty, n, n^{\prime}\right) & =2|\lambda| \varphi_{-}\left(\frac{1}{2|\lambda|}, n^{\prime}-1\right), \\
\phi_{1}\left(0, \infty, n, n^{\prime}\right) & =\min \left\{n, n^{\prime}\right\}, \\
\phi_{2}\left(0, \infty, n, n^{\prime}\right) & =\min \left\{n, n^{\prime}\right\}-1,
\end{aligned}
$$

in which $\lambda, \lambda^{\prime} \notin\{0, \infty\}$, and $\gamma:=\min \left\{\left|\frac{\lambda^{\prime}-\lambda}{\lambda}\right|,\left|\frac{\overline{\lambda^{\prime}}-\lambda}{\lambda}\right|\right\}, \gamma^{\prime}:=\min \left\{\left|\frac{\lambda^{\prime}-\lambda}{\lambda^{\prime}}\right|,\left|\frac{\overline{\lambda^{\prime}}-\lambda}{\lambda^{\prime}}\right|\right\}$.

The proof of Lemma 3.1 is long, quite technical, and provides no further insight, so we defer it to A. 


\subsection{The main result}

In the following, we will present the relative perturbation theory for Hermitian matrix pairs, using the above results. To derive our bounds we need to specify what assumptions we will use within this paper.

We consider two Hermitian matrix pairs $(A, B)$ and $(\widetilde{A}, \widetilde{B})$, and two nonsingular matrices $X=$ $\left[\begin{array}{ll}X_{1} & X_{2}\end{array}\right]$ and $\widetilde{X}=\left[\begin{array}{ll}\widetilde{X}_{1} & \widetilde{X}_{2}\end{array}\right]$ for which the following assumptions hold:

(A1) Both matrix pairs are regular.

(A2) $X, \widetilde{X}$ are the matrices to canonicalize the unperturbed and perturbed matrix pairs with special partitions. In detail, they satisfy the following statements:

(a) $X^{H}(A, B) X=(\Lambda, \Omega)=\left(\Lambda_{1}, \Omega_{1}\right) \oplus\left(\Lambda_{2}, \Omega_{2}\right)$, or equivalently, $X_{i}^{H} A X_{i}=\Lambda_{i}, X_{i}^{H} B X_{i}=$ $\Omega_{i}, i=1,2$ and $X_{1}^{H} A X_{2}=X_{1}^{H} B X_{2}=0$, where $\left(\Lambda_{i}, \Omega_{i}\right), i=1,2$ are of the form (2.2), namely

$$
\left(\Lambda_{i}, \Omega_{i}\right)=\bigoplus_{j_{i}=1}^{m_{i}}\left(\Lambda_{i, j_{i}}, \Omega_{i, j_{i}}\right), i=1,2,
$$

where $\left(\Lambda_{i, j_{i}}, \Omega_{i, j_{i}}\right)$ are blocks of size $n_{i, j_{i}}$ as in (2.2) with corresponding eigenvalue $\lambda_{i, j_{i}}$.

(b) Similarly, $\widetilde{X}^{H}(\widetilde{A}, \widetilde{B}) \widetilde{X}=(\widetilde{\Lambda}, \widetilde{\Omega})=\left(\widetilde{\Lambda}_{1}, \widetilde{\Omega}_{1}\right) \oplus\left(\widetilde{\Lambda}_{2}, \widetilde{\Omega}_{2}\right)$, or equivalently, $\widetilde{X}_{i}^{H} \widetilde{A} \widetilde{X}_{i}=$ $\widetilde{\Lambda}_{i}, \widetilde{X}_{i}^{H} \widetilde{B} \widetilde{X}_{i}=\widetilde{\Omega}_{i}, i=1,2$ and $\widetilde{X}_{1}^{H} \widetilde{A} \widetilde{X}_{2}=\widetilde{X}_{1}^{H} \widetilde{B}_{X_{2}}=0$, where $\left(\widetilde{\Lambda}_{i}, \widetilde{\Omega}_{i}\right), i=1,2$ are of the form (2.2), namely

$$
\left(\widetilde{\Lambda}_{i}, \widetilde{\Omega}_{i}\right)=\bigoplus_{\tilde{j}_{i}=1}^{\widetilde{m}_{i}}\left(\widetilde{\Lambda}_{i, \tilde{j}_{i}}, \widetilde{\Omega}_{i, \widetilde{j}_{i}}\right), i=1,2
$$

where $\left(\widetilde{\Lambda}_{i, \widetilde{j}_{i}}, \widetilde{\Omega}_{i, \widetilde{j}_{i}}\right)$ are blocks of size $\widetilde{n}_{i, \widetilde{j}_{i}}$ as in (2.2) with corresponding eigenvalue $\widetilde{\lambda}_{i, j_{i}}$.

(c) $\Lambda_{i}, \Omega_{i}, \widetilde{\Lambda}_{i}, \widetilde{\Omega}_{i}$ are of the same size for $i=1,2$, which means that $X_{i}, \widetilde{X}_{i}$ are of the same size for $i=1,2$, too. Note that this only means $\sum_{j_{i}=1}^{m_{i}} n_{i, j_{i}}=\sum_{\widetilde{j}_{i}=1}^{\widetilde{m}_{i}} \widetilde{n}_{i, \widetilde{j}_{i}}$ rather than any further assumption on $m_{i}, n_{i, j_{i}}, \widetilde{m}_{i}, \widetilde{n}_{i, \widetilde{j}_{i}}$.

(A3) It holds that

$$
\begin{array}{ll}
\lambda\left(\Lambda_{1}, \Omega_{1}\right) \cap \lambda\left(\Lambda_{2}, \Omega_{2}\right)=\emptyset, & \lambda\left(\widetilde{\Lambda}_{1}, \widetilde{\Omega}_{1}\right) \cap \lambda\left(\widetilde{\Lambda}_{2}, \widetilde{\Omega}_{2}\right)=\emptyset, \\
\lambda\left(\Lambda_{1}, \Omega_{1}\right) \cap \lambda\left(\widetilde{\Lambda}_{2}, \widetilde{\Omega}_{2}\right)=\emptyset, & \lambda\left(\Lambda_{2}, \Omega_{2}\right) \cap \lambda\left(\widetilde{\Lambda}_{1}, \widetilde{\Omega}_{1}\right)=\emptyset .
\end{array}
$$

(A4) It holds that

$$
\infty \notin \lambda\left(\Lambda_{1}, \Omega_{1}\right), \quad 0 \notin \lambda\left(\Lambda_{2}, \Omega_{2}\right), \quad \infty \notin \lambda\left(\widetilde{\Lambda}_{1}, \widetilde{\Omega}_{1}\right), \quad 0 \notin \lambda\left(\widetilde{\Lambda}_{2}, \widetilde{\Omega}_{2}\right),
$$

which implies $\Omega_{1}, \widetilde{\Omega}_{1}, \Lambda_{2}, \widetilde{\Lambda}_{2}$ are nonsingular. 
Remark 3.1. Let us say something more to illustrate the practical meaning of such complicated assumptions. Clearly, (A1) is necessary.

By (A2) we partition the whole space into two (eigen-)subspaces $X_{1}, X_{2}$, such that $X_{1}$ makes $(A, B)$ to behave as $\left(\Lambda_{1}, \Omega_{1}\right)$ and $X_{2}$ makes $(A, B)$ to behave as $\left(\Lambda_{2}, \Omega_{2}\right)$. Without loss of generality, we may call the former one "focused" and the latter one "ignored". Similarly to this, for perturbed case we partition the whole space into two (eigen-)subspaces $\widetilde{X}_{1}, \widetilde{X}_{2}$ such that one makes $(\widetilde{A}, \widetilde{B})$ to behave as $\left(\widetilde{\Lambda}_{1}, \widetilde{\Omega}_{1}\right)$ and other makes $(\widetilde{A}, \widetilde{B})$ to behave as $\left(\widetilde{\Lambda}_{2}, \widetilde{\Omega}_{2}\right)$. We hope to observe the effect of the perturbation, so $X_{1}, \widetilde{X}_{1}$ should be related and very close to each other if the perturbation is small enough. As a result $\Lambda_{1}, \widetilde{\Lambda}_{1}$ are related, and so do $\Omega_{1}, \widetilde{\Omega}_{1}$, and also holds for the ignored subspaces.

(A3) is a mathematical form of the practical requirement: the eigenvalues in the focused subspaces and the ignored subspaces may not be the same or close, otherwise we cannot distinguish the effects from the focused and ignored subspaces.

(A4) is a technical assumption: we focus the possible eigenvalue 0 and ignore the possible eigenvalue $\infty$. Under these assumption, the purpose of the perturbation analysis is to find a quantitative description how close the eigenvalues and eigenspaces of the unperturbed and perturbed matrix pairs are.

Here is another implicit technical requirement that we will use later. If a complex eigenvalue is focused, then by the blocks of type R4, its conjugate, whish is also an eigenvalue, is focused, too. It is quite possible that the related eigenvalue of the perturbed matrix pair is also complex. We would have to measure how close two conjugate-closed complex number pairs are, and a natural way is to measure the complex unperturbed and perturbed eigenvalues with non-negative (or non-positive) imaginary parts.

Now we can state the central theorem of this paper, namely Theorem 3.1.

Theorem 3.1. Let $(A, B)$ and $(\widetilde{A}, \widetilde{B})=(A+\delta A, B+\delta B)$ be regular Hermitian matrix pairs and $X$ and $\widetilde{X}$ be nonsingular matrices. If the assumptions (A1)-(A4) hold for the perturbed and unperturbed matrix pairs, then we have

$$
\begin{aligned}
\left\|\sin \Theta\left(\operatorname{span}\left(X_{1}\right), \operatorname{span}\left(\widetilde{X}_{1}\right)\right)\right\|_{F} \leq \phi_{1}^{\max } & \kappa_{2}(X) \kappa_{2}(\widetilde{X})\left\|A^{+} \delta A\right\|_{F} \\
& +\phi_{2}^{\max }\|X\|_{2}^{2}\left\|\widetilde{X}^{-1}\right\|_{2}^{2}\left\|\widetilde{B}^{+} \delta B\right\|_{F},
\end{aligned}
$$

where

$$
\phi_{1}^{\max }:=\max _{j_{1}, j_{2}} \phi_{1}\left(\widetilde{\lambda}_{1, j_{1}}, \lambda_{2, j_{2}}, \widetilde{n}_{1, j_{1}}, n_{2, j_{2}}\right), \quad \phi_{2}^{\max }:=\max _{j_{1}, j_{2}} \phi_{2}\left(\widetilde{\lambda}_{1, j_{1}}, \lambda_{2, j_{2}}, \widetilde{n}_{1, j_{1}}, n_{2, j_{2}}\right),
$$

and $\phi_{1}(\cdot, \cdot, \cdot, \cdot), \phi_{2}(\cdot, \cdot, \cdot, \cdot)$ are defined in (3.2). Moreover, if $B$ is nonsingular,

$$
\left\|\sin \Theta\left(\operatorname{span}\left(X_{1}\right), \operatorname{span}\left(\widetilde{X}_{1}\right)\right)\right\|_{F} \leq \kappa_{2}(X) \kappa_{2}(\widetilde{X})\left(\phi_{1}^{\max }\left\|A^{+} \delta A\right\|_{F}+\phi_{2}^{\max }\left\|B^{-1} \delta B\right\|_{F}\right) .
$$

Proof. First, similarly to the discussion in [29, Lemma 2], using $Q R$ decomposition of the matrices $B X_{2}$ and $\widetilde{X}_{1}$ and the fact that $\left\|\sin \Theta\left(X_{1}, \widetilde{X}_{1}\right)\right\|=\left\|Q_{2}^{*} \widetilde{Q}_{1}\right\|$, where $Q_{2}$ and $\widetilde{Q}_{1}$ form the orthonormal basis for $\operatorname{span}\left(X_{2}\right)$ and $\operatorname{span}\left(\widetilde{X}_{1}\right)$, we observe that

$$
\left\|\sin \Theta\left(\operatorname{span}\left(X_{1}\right), \operatorname{span}\left(\widetilde{X}_{1}\right)\right)\right\|_{F} \leq\left\|\left(B X_{2}\right)^{+}\right\|_{2}\left\|\widetilde{X}_{1}^{+}\right\|_{2}\|\| X_{2}^{H} B \widetilde{X}_{1} \|_{F}
$$


From $B X_{2}=X^{-H}\left[\begin{array}{c}0 \\ \Omega_{2}\end{array}\right]$, we get $\left\|B X_{2}\right\|_{2} \leq\left\|X^{-1}\right\|_{2}$ and $\left\|\left(B X_{2}\right)^{+}\right\|_{2}=\left\|\left[\begin{array}{ll}0 & \Omega_{2}^{H}\end{array}\right]^{+} X\right\|_{2} \leq$ $\|X\|_{2}$. Similarly, $\left\|\widetilde{B} \widetilde{X}_{1}\right\|_{2} \leq\left\|\widetilde{X}^{-1}\right\|_{2}$.

Then, to bound $\left\|\sin \Theta\left(\operatorname{span}\left(X_{1}\right), \operatorname{span}\left(\widetilde{X}_{1}\right)\right)\right\|_{F}$, we need to estimate $\left\|X_{2}^{H} B \widetilde{X}_{1}\right\|_{F}$. Using (3.3), define $L, R$ by:

$$
L=L_{1} \oplus L_{2}=\bigoplus_{i=1}^{2} \bigoplus_{j_{i}=1}^{m_{i}} L_{i, j_{i}}, \quad R=R_{1} \oplus R_{2}=\bigoplus_{i=1}^{2} \bigoplus_{j_{i}=1}^{m_{i}} R_{i, j_{i}},
$$

where $L_{i, j_{i}}=\Lambda_{i, j_{i}} \Omega_{i, j_{i}}, R_{i, j_{i}}=I$ if the corresponding block pair is of type $\mathbf{R} \mathbf{1}$ or $L_{i, j_{i}}=I, R_{i, j_{i}}=$ $\Omega_{i, j_{i}} \Lambda_{i, j_{i}}$ if not. Here the size of $I$ is determined under the condition that $L_{i, j_{i}}$ and $R_{i, j_{i}}$ are of the same size. Then, it is easy to check that $L$ and $R$ are lower triangular matrices since $\Lambda_{i, j_{i}}$ and $\Omega_{i, j_{i}}$ are matrices from the pairs of the form (2.2). Also $L_{1}=I$ by (A4). Note that if $L_{i, j_{i}}=\Lambda_{i, j_{i}} \Omega_{i, j_{i}}, R_{i, j_{i}}=I$, when the corresponding block pair is of type $\mathbf{R} 1$, then it is easy to see that $\Lambda_{i, j_{i}} \Lambda_{i, j_{i}}=I$ and we have $\Lambda L=\Omega=\Omega R$. Also, in second case when $L_{i, j_{i}}=I, R_{i, j_{i}}=\Omega_{i, j_{i}} \Lambda_{i, j_{i}}$, it will be that $\Omega_{i, j_{i}} \Omega_{i, j_{i}}=I$ and $\Omega R=\Lambda=\Lambda L$. Thus, we can conclude that

$$
X^{H} A X L=\Lambda L=\Omega R=X^{H} B X R
$$

and then

$$
A X L=B X R, \text { and similarly } \widetilde{A} \widetilde{X} \widetilde{L}=\widetilde{B} \widetilde{X} \widetilde{R} .
$$

Also, $\widetilde{L}$ and $\widetilde{R}$ are lower triangular matrices and $\widetilde{L}_{1}=I$. Then

$$
\begin{aligned}
R_{2}^{H} X_{2}^{H} B \widetilde{X}_{1} \widetilde{L}_{1} & -L_{2}^{H} X_{2}^{H} B \widetilde{X}_{1} \widetilde{R}_{1} \\
& =R_{2}^{H} X_{2}^{H} B \widetilde{X}_{1} \widetilde{L}_{1}-L_{2}^{H} X_{2}^{H} \widetilde{B} \widetilde{X}_{1} \widetilde{R}_{1}+L_{2}^{H} X_{2}^{H} \delta B \widetilde{X}_{1} \widetilde{R}_{1} \\
& =L_{2}^{H} X_{2}^{H} A \widetilde{X}_{1} \widetilde{L}_{1}-L_{2}^{H} X_{2}^{H} \widetilde{A} \widetilde{X}_{1} \widetilde{L}_{1}+L_{2}^{H} X_{2}^{H} \delta B \widetilde{X}_{1} \widetilde{R}_{1} \\
& =-L_{2}^{H} X_{2}^{H} \delta A \widetilde{X}_{1} \widetilde{L}_{1}+L_{2}^{H} X_{2}^{H} \delta B \widetilde{X}_{1} \widetilde{R}_{1} .
\end{aligned}
$$

Note that $A X_{2}=X^{-H}\left[\begin{array}{c}0 \\ \Lambda_{2}\end{array}\right]$. Since $\Lambda_{2}$ is nonsingular, $A X_{2}$ has full column rank, which implies that $\operatorname{span}\left(X_{2}\right) \cap \mathcal{N}(A)=\emptyset$, where $\mathcal{N}(A)$ is the nullspace of $A$. Note that $I-A^{+} A$ is the orthogonal projector onto $\mathcal{N}(A)$. Thus, $A^{+} A X_{2}=X_{2}$, whence

$$
\begin{aligned}
R_{2}^{H} X_{2}^{H} B \widetilde{X}_{1} \widetilde{L}_{1}-L_{2}^{H} X_{2}^{H} B \widetilde{X}_{1} \widetilde{R}_{1} & =-L_{2}^{H} X_{2}^{H} A A^{+} \delta A \widetilde{X}_{1} \widetilde{L}_{1}+L_{2}^{H} X_{2}^{H} \delta B \widetilde{X}_{1} \widetilde{R}_{1} \\
& =-R_{2}^{H} X_{2}^{H} B A^{+} \delta A \widetilde{X}_{1} \widetilde{L}_{1}+L_{2}^{H} X_{2}^{H} \delta B \widetilde{X}_{1} \widetilde{R}_{1} .
\end{aligned}
$$

To estimate the norm of the solution $X_{2}^{H} B \widetilde{X}_{1}$ to the structured Sylvester equation (3.9), we will use the fact that the operator $Y \mapsto R_{2}^{H} Y \widetilde{L}_{1}-L_{2}^{H} Y \widetilde{R}_{1}$ is linear. Hence, if $Y^{(1)}$ is the solution to

$$
R_{2}^{H} Y \widetilde{L}_{1}-L_{2}^{H} Y \widetilde{R}_{1}=-R_{2}^{H} X_{2}^{H} B A^{+} \delta A \widetilde{X}_{1} \widetilde{L}_{1}
$$

and $Y^{(2)}$ is the solution to

$$
R_{2}^{H} Y \widetilde{L}_{1}-L_{2}^{H} Y \widetilde{R}_{1}=L_{2}^{H} X_{2}^{H} \delta B \widetilde{X}_{1} \widetilde{R}_{1}
$$


then $X_{2}^{H} B \widetilde{X}_{1}=Y^{(1)}+Y^{(2)}$ is the solution to (3.9). Thus estimating the norms of the two $Y^{(i)}$ and then using $\left\|X_{2}^{H} B \widetilde{X}_{1}\right\| \leq\left\|Y^{(1)}\right\|+\left\|Y^{(2)}\right\|$ (for any norm) will yield the result.

For this, first we consider $Y^{(1)}$ and (3.10), related to $\delta B=0$ in (3.9), i.e., for $j_{1}=1, \ldots, m_{1}$ and $j_{2}=1, \ldots, m_{2}$ :

$$
R_{2, j_{2}}^{H} Y_{j_{2}, j_{1}}^{(1)} \widetilde{L}_{1, j_{1}}-L_{2, j_{2}}^{H} Y_{j_{2}, j_{1}}^{(1)} \widetilde{R}_{1, j_{1}}=-R_{2, j_{2}}^{H} X_{2, j_{2}}^{H} B A^{+} \delta A \widetilde{X}_{1, j_{1}} \widetilde{L}_{1, j_{1}} .
$$

Noticing $\widetilde{L}_{1}=I$, this equation in $Y_{j_{2}, j_{1}}^{(1)}$ is of the form (3.1), i.e., of the form (3.1a) or (3.1b) depending on the type of corresponding block pairs in (3.3). By Lemma 3.1,

$$
\left\|Y_{j_{2}, j_{1}}^{(1)}\right\|_{F} \leq \phi_{1}\left(\widetilde{\lambda}_{1, j_{1}}, \lambda_{2, j_{2}}, \widetilde{n}_{1, j_{1}}, n_{2, j_{2}}\right)\left\|X_{2, j_{2}}^{H} B A^{+} \delta A \widetilde{X}_{1, j_{1}}\right\|_{F}
$$

Then

$$
\begin{aligned}
\left\|Y^{(1)}\right\|_{F} & =\sqrt{\sum_{j_{1}=1}^{m_{1}} \sum_{j_{2}=1}^{m_{2}}\left\|Y_{j_{2}, j_{1}}^{(1)}\right\|_{F}^{2}} \\
& \leq \max _{j_{1}, j_{2}} \phi_{1}\left(\widetilde{\lambda}_{1, j_{1}}, \lambda_{2, j_{2}}, \widetilde{n}_{1, j_{1}}, n_{2, j_{2}}\right) \sqrt{\sum_{j_{1}=1}^{m_{1}} \sum_{j_{2}=1}^{m_{2}}\left\|X_{2, j_{2}}^{H} B A^{+} \delta A \widetilde{X}_{1, j_{1}}\right\|_{F}^{2}} \\
& \leq \max _{j_{1}, j_{2}} \phi_{1}\left(\widetilde{\lambda}_{1, j_{1}}, \lambda_{2, j_{2}}, \widetilde{n}_{1, j_{1}}, n_{2, j_{2}}\right)\left\|X_{2}^{H} B A^{+} \delta A \widetilde{X}_{1}\right\|_{F} \\
& \leq \max _{j_{1}, j_{2}} \phi_{1}\left(\widetilde{\lambda}_{1, j_{1}}, \lambda_{2, j_{2}}, \widetilde{n}_{1, j_{1}}, n_{2, j_{2}}\right)\left\|B X_{2}\right\|_{2}\left\|\widetilde{X}_{1}\right\|_{2}\left\|A^{+} \delta A\right\|_{F} \\
& \leq \max _{j_{1}, j_{2}} \phi_{1}\left(\widetilde{\lambda}_{1, j_{1}}, \lambda_{2, j_{2}}, \widetilde{n}_{1, j_{1}}, n_{2, j_{2}}\right)\left\|X^{-1}\right\|_{2}\|\widetilde{X}\|_{2}\left\|A^{+} \delta A\right\|_{F} .
\end{aligned}
$$

For $Y^{(2)}$, which is solution of (3.11), related to $\delta A=0$ in (3.9), we obtain

$$
\left\|Y^{(2)}\right\|_{F} \leq \max _{j_{1}, j_{2}} \phi_{2}\left(\widetilde{\lambda}_{1, j_{1}}, \lambda_{2, j_{2}}, \widetilde{n}_{1, j_{1}}, n_{2, j_{2}}\right)\left\|X_{2}^{H} \delta B \widetilde{X}_{1}\right\|_{F}
$$

In case where $B$ is nonsingular,

$$
\left\|Y^{(2)}\right\|_{F} \leq \max _{j_{1}, j_{2}} \phi_{2}\left(\widetilde{\lambda}_{1, j_{1}}, \lambda_{2, j_{2}}, \widetilde{n}_{1, j_{1}}, n_{2, j_{2}}\right)\left\|X^{-1}\right\|_{2}\|\widetilde{X}\|_{2}\left\|B^{-1} \delta B\right\|_{F}
$$

Thus (3.7) holds.

For the general case, since $\widetilde{\Omega}_{1}$ is nonsingular, $\widetilde{B}^{+} \widetilde{B} \widetilde{X}_{1}=\widetilde{X}_{1}$. Then

$$
\left\|Y^{(2)}\right\|_{F} \leq \max _{j_{1}, j_{2}} \phi_{2}\left(\widetilde{\lambda}_{1, j_{1}}, \lambda_{2, j_{2}}, \widetilde{n}_{1, j_{1}}, n_{2, j_{2}}\right)\|X\|_{2}\left\|\widetilde{X}^{-1}\right\|_{2}\left\|\widetilde{B}^{+} \delta B\right\|_{F},
$$

so (3.5) holds.

Let us illustrate the quality of bounds (3.5) and (3.7) with the following small example. 
Example 3.1. Consider a regular matrix pair $(A, B)$, where

$$
A=\left[\begin{array}{llll}
0 & 8 & \varrho & 2 \\
8 & 0 & 2 & 0 \\
\varrho & 2 & 0 & 8 \\
2 & 0 & 8 & 0
\end{array}\right] \text { and } B=\left[\begin{array}{cccc}
0 & 0 & 0 & 1 \\
0 & 0 & 1 & 0 \\
0 & 1 & 0 & 0 \\
1 & 0 & 0 & 0
\end{array}\right]
$$

where $|\varrho| \leq 1$. The corresponding perturbed pair $(\widetilde{A}, \widetilde{B})$ is given as $\widetilde{A}=A+\xi \delta A$ and $\widetilde{B}=$ $B+\xi \delta B$, where $\xi=10^{-8}$ and $\delta A, \delta B$ are random Hermitian matrices with elements uniformly distributed in interval $(0,1)$. We will analyze the quality of the bound (3.1) for a different choices of the value $\varrho$ repeated a 100 times. The maximal and the minimal values of the quantities which appear in the bound (3.1) for 100 generated perturbations $(\delta A, \delta B)$ are given in the Table 3.1.

\begin{tabular}{|l|c|c|c|c|l|l|l|}
\hline \multirow{2}{*}{$\varrho$} & \multicolumn{2}{|c|}{ Exact $\|\sin \Theta\|_{F}$} & \multicolumn{2}{c|}{ Bound $(3.1)$} & $\kappa_{2}(X)$ & \multicolumn{2}{c|}{$\kappa_{2}(\tilde{X})$} \\
\cline { 2 - 8 } & $\min$ & $\max$ & $\min$ & $\max$ & & $\min$ & $\max$ \\
\hline 0 & $5.4812 \cdot 10^{-10}$ & $2.8099 \cdot 10^{-9}$ & $5.4311 \cdot 10^{-8}$ & $2.0468 \cdot 10^{-6}$ & 1 & 1.0766 & 16.770 \\
$10^{-14}$ & $1.5450 \cdot 10^{-10}$ & $2.7125 \cdot 10^{-9}$ & $4.0485 \cdot 10^{-7}$ & $6.5515 \cdot 10^{-6}$ & 9.7053 & 1.0928 & 13.687 \\
1 & $6.2533 \cdot 10^{-10}$ & $3.0239 \cdot 10^{-9}$ & $3.1436 \cdot 10^{-4}$ & $8.6771 \cdot 10^{-3}$ & 1 & $6.9469 \cdot 10^{3}$ & $2.1380 \cdot 10^{5}$ \\
\hline
\end{tabular}

Table 3.1: Comparison of the exact value for $\left\|\sin \Theta\left(\operatorname{span}\left(X_{1}\right), \operatorname{span}\left(\widetilde{X}_{1}\right)\right)\right\|_{F}$ and the bound (3.1).

For the case when $\varrho=0$ eigenvalues -6 and 10 are both semi-simple and in that case our bound gives the best result because the conditions of the subspace matrices $X$ and $\widetilde{X}$ are small since in both cases subspaces are spanned by linear independent eigenvectors.

Note, that for $\varrho \neq 0$ the eigenvalues of the pair $(A, B)$ correspond with the following Jordan form

$$
J=\operatorname{diag}\left\{\left[\begin{array}{cc}
-6 & 1 \\
0 & -6
\end{array}\right],\left[\begin{array}{cc}
10 & 1 \\
0 & 10
\end{array}\right]\right\} .
$$

In that case, if $\varrho=1$, in 75 of 100 cases our bound is of the size $10^{-4}$, which is acceptable since the condition number the matrix $\widetilde{X}$ vary between $7 \cdot 10^{3}$ and $2.2 \cdot 10^{5}$. It is important to emphasize that in the presence of Jordan form the bound (3.1) is given for the subspaces spanned by the columns of $X_{1}=X(:, 1: 2)$ and $\widetilde{X}_{1}=\widetilde{X}(:, 1: 2)$. The columns of $X_{1}$ contain one eigenvector and one generalized eigenvector, respectively, which correspond with double unperturbed eigenvalue from the Jordan form, while the columns of $\widetilde{X}_{1}$ contain two eigenvectors which correspond with the perturbed eigenvalues which are simple, since under the small perturbation perturbed pair lose the Jordan form. A similar example, but for a regular quadratic eigenvalue problem, will be given in the last section (Example 5.3).

An interesting situation occurs, for a small $\varrho$, for example $\varrho=10^{-14}$. In that case, the rounding error in calculation of the eigenvectors (for example using MATLAB function eig) for the matrix pair $(A, B)$ will give four linear independent eigenvectors in $X$ and $\widetilde{X}$ and Jordan form will not be detected. In that case our bound is again acceptable, and give us good information about size of the perturbation.

Also, a more general but weaker result can be given for any unitarily invariant norm, as is shown in Theorem 3.2. 
Theorem 3.2. Given a Hermitian matrix pair $(A, B)$ and its corresponding perturbed pair $(\widetilde{A}, \widetilde{B})$ with $B, \widetilde{B}$ nonsingular (which guarantees assumption (A1)). Under the assumptions (A2)-(A4), if there exist $\alpha \geq 0$ and $\delta>0$ such that

$$
\begin{array}{ll}
\left\|\Omega_{2} \Lambda_{2}\right\|_{2} \leq \alpha, & \left\|\left(\widetilde{\Omega}_{1} \widetilde{\Lambda}_{1}\right)^{-1}\right\|_{2}^{-1} \geq \alpha+\delta, \quad \text { or } \\
\left\|\left(\Omega_{2} \Lambda_{2}\right)^{-1}\right\|_{2}^{-1} \geq \alpha+\delta, & \left\|\widetilde{\Omega}_{1} \widetilde{\Lambda}_{1}\right\|_{2} \leq \alpha,
\end{array}
$$

then, for any unitarily invariant norm $\|\cdot\|_{\text {ui }}$ and $p, q$ where $p^{-1}+q^{-1}=1$,

$$
\left\|\sin \Theta\left(\operatorname{span}\left(X_{1}\right), \operatorname{span}\left(\widetilde{X}_{1}\right)\right)\right\|_{\mathrm{ui}} \leq \mu \kappa_{2}(X) \kappa_{2}(\widetilde{X}) \sqrt[q]{\left\|A^{+} \delta A\right\|_{\mathrm{ui}}^{q}+\left\|B^{-1} \delta B\right\|_{\mathrm{ui}}^{q}},
$$

where $\mu=\frac{\delta}{\sqrt[p]{\alpha^{p}+(\alpha+\delta)^{p}}}$. Also, also

$$
\left\|\sin \Theta\left(\operatorname{span}\left(X_{1}\right), \operatorname{span}\left(\widetilde{X}_{1}\right)\right)\right\|_{\mathrm{ui}} \leq \kappa_{2}(X) \kappa_{2}(\widetilde{X})\left(\frac{\left\|A^{+} \delta A\right\|_{\mathrm{ui}}}{\frac{\delta}{\alpha}}+\frac{\left\|B^{-1} \delta B\right\|_{\mathrm{ui}}}{\frac{\delta}{\alpha+\delta}}\right) .
$$

Proof. The proof is similar to the proof of Theorem 3.1, except a modified version of [22, Lemma 2.3] is used to estimate (3.9) rather than Lemma 3.1. The "modified" version is to get rid of the assumption " $\Omega, \Gamma$ are Hermitian". That assumption appears there for using [22, Lemma 2.2] to make it clear that (3.9) has a unique solution. But now this is guaranteed by Lemma 3.1.

Remark 3.2. Theorem 3.2 is not that useful in general. If there is no semi-simple eigenvalue, we have the bounds

$$
\left\|\Omega_{2} \Lambda_{2}\right\|_{2} \leq 2 \max _{i_{2}}\left|\lambda_{2, i_{2}}\right|, \quad\left\|\left(\widetilde{\Omega}_{1} \widetilde{\Lambda}_{1}\right)^{-1}\right\|_{2} \leq \max _{\widetilde{i}_{1}} n_{\widetilde{i}_{1}}\left|\widetilde{\lambda}_{1, \widetilde{i}_{1}}^{-1}\right| .
$$

This means that the gap must be bigger than $\left(2-\frac{1}{2}\right) \alpha+2 \delta$, which is not the expected one and it would be $k \delta$ for some scalar $k$. This theorem is good only for the case that all eigenvalues are semi-simple.

Besides, if only one eigenvalue is considered, we obtain the eigenvalue perturbation bounds in Theorem 3.3.

Theorem 3.3. Let $(A, B)$ and $(\widetilde{A}, \widetilde{B})=(A+\delta A, B+\delta B)$ be regular Hermitian matrix pairs where $B, \widetilde{B}$ are nonsingular, and let $X$ and $\widetilde{X}$ be nonsingular matrices.

Suppose that $X=\left[\begin{array}{lll}X_{1} & \ldots & X_{m}\end{array}\right]$ is the matrix to canonicalize the unperturbed matrix pair with special partitions, namely $X^{H}(A, B) X=(\Lambda, \Omega)=\bigoplus_{j=1}^{m_{i}}\left(\Lambda_{j}, \Omega_{j}\right)$, or equivalently, $X_{j}^{H} A X_{j}=\Lambda_{j}, X_{j}^{H} B X_{j}=\Omega_{j}, j=1, \ldots, m$ and $X_{j}^{H} A X_{j^{\prime}}=X_{j}^{H} B X_{j^{\prime}}=0, j \neq j^{\prime}$, where $\left(\Lambda_{j}, \Omega_{j}\right)$ are blocks of size $n_{j}$ if the corresponding eigenvalue is real or $2 n_{j}$ if the corresponding eigenvalue is imaginary as in (2.2) with corresponding eigenvalue $\lambda_{j}$. Analogously $\widetilde{X}=\left[\begin{array}{lll}\widetilde{X}_{1} & \ldots & \widetilde{X}_{\widetilde{m}}\end{array}\right]$, and $\widetilde{\Lambda}_{\tilde{j}}, \widetilde{\Omega}_{\tilde{j}}, \widetilde{n}_{\tilde{j}}, \widetilde{\lambda}_{\tilde{j}}, \widetilde{m}$ play similar roles for $(\widetilde{A}, \widetilde{B})$.

Suppose that $\lambda \in \lambda\left(\Lambda_{j}, \Omega_{j}\right), \widetilde{\lambda} \in \lambda\left(\widetilde{\Lambda}_{\tilde{j}}, \widetilde{\Omega}_{\tilde{j}}\right)$, where $\lambda, \widetilde{\lambda}$ are nonzero and lie in the upper half plane. Let $\gamma=\left|\frac{\widetilde{\lambda}-\lambda}{\lambda}\right|, \widetilde{\gamma}=\left|\frac{\widetilde{\lambda}-\lambda}{\widetilde{\lambda}}\right|$. Then, 
1. provided $\gamma \leq \frac{5-\sqrt{17}}{4}, \widetilde{\gamma} \leq \frac{5-\sqrt{17}}{4}$,

$$
\frac{\gamma^{n_{j}} \widetilde{\gamma}^{\widetilde{n}_{\tilde{j}}}}{\sqrt{\gamma^{2}+\widetilde{\gamma}^{2}}} \leq 2 \frac{\left\|X^{-1}\right\|_{2}\|\widetilde{X}\|_{2}}{\left\|X_{j}^{H} B \widetilde{X}_{\tilde{j}}\right\|_{F}} \sqrt{\left(\begin{array}{c}
\widetilde{n}_{\tilde{j}}+n_{j}-1 \\
n_{j}
\end{array}\right)^{2}\left\|A^{+} \delta A\right\|_{F}^{2}+\left(\begin{array}{c}
\widetilde{n}_{\tilde{j}}+n_{j}-1 \\
\widetilde{n}_{\tilde{j}}
\end{array}\right)^{2}\left\|B^{-1} \delta B\right\|_{F}^{2}} ;
$$

2. Specially, if $n_{j}=\widetilde{n}_{\tilde{j}}=1$ and $\left|x^{H} B \widetilde{x}\right|>\left\|X^{-1}\right\|_{2}\|\widetilde{X}\|_{2}\left\|B^{-1} \delta B\right\|_{\text {ui }}$, where $x, \widetilde{x}$ are the corresponding eigenvectors of $\lambda, \widetilde{\lambda}$ respectively, we have

$$
\gamma \leq \frac{\left\|X^{-1}\right\|_{2}\|\widetilde{X}\|_{2}\left(\left\|A^{+} \delta A\right\|_{\mathrm{ui}}+\left\|B^{-1} \delta B\right\|_{\mathrm{ui}}\right)}{\left|x^{H} B \widetilde{x}\right|-\left\|X^{-1}\right\|_{2}\|\widetilde{X}\|_{2}\left\|B^{-1} \delta B\right\|_{\mathrm{ui}}}
$$

Proof. Using (3.3), define $R$ by $R=\bigoplus_{j=1}^{m} R_{j}$, where $R_{j}=\Omega_{j} \Lambda_{j}$, which is similar to that in the proof of Theorem 3.1. By (3.8), similarly to the discussion in the proof of Theorem 3.1, we have

$$
\begin{aligned}
R^{H} X^{H} B \widetilde{X}-X^{H} B \widetilde{X} \widetilde{R} & =-X^{H} \delta A \widetilde{X}+X^{H} \delta B \widetilde{X} \widetilde{R} \\
& =-R^{H} X^{H} B A^{+} \delta A \widetilde{X}+X^{H} \delta B \widetilde{X} \widetilde{R} .
\end{aligned}
$$

Then, considering each block, for $j=1, \ldots, m$ and $\widetilde{j}=1, \ldots, \widetilde{m}$,

$$
R_{j}^{H} X_{j}^{H} B \widetilde{X}_{\tilde{j}}-X_{j}^{H} B \widetilde{X}_{\tilde{j}} \widetilde{R}_{\tilde{j}}=-R_{j}^{H} X_{j}^{H} B A^{+} \delta A \widetilde{X}_{\tilde{j}}+X_{j}^{H} \delta B \widetilde{X}_{\tilde{j}} \widetilde{R}_{\tilde{j}} .
$$

By $B X=X^{-H} \cdot\left(\bigoplus_{j=1}^{m} F_{n_{j}}\right)$,

$$
R_{j}^{H} X_{j}^{H} B \widetilde{X}_{\widetilde{j}}-X_{j}^{H} B \widetilde{X}_{\widetilde{j}} \widetilde{R}_{\widetilde{j}}=-R_{j}^{H} \widehat{F}_{n_{j}} X^{-1} A^{+} \delta A \widetilde{X}_{\widetilde{j}}+\widehat{F}_{n_{j}} X^{-1} B^{-1} \delta B \widetilde{X}_{\widetilde{j}} \widetilde{R}_{\widetilde{j}},
$$

where $\widehat{F}_{n_{j}}=X^{H} B X_{j}=\left[\begin{array}{c}\vdots \\ F_{n_{j}} \\ \vdots\end{array}\right]$, the matrix consisting of $F_{n_{j}}$ and possible zero blocks upper and lower.

Since $X_{j}^{H} B \widetilde{X}_{\widetilde{j}}$ is the solution to the structured Sylvester equation (3.12), which is of the form (3.1a), by Lemma 3.1, we get

$$
\left\|X_{j}^{H} B \widetilde{X}_{\tilde{j}}\right\|_{F} \leq \phi_{1}\left\|\widehat{F}_{n_{j}} X^{-1} A^{+} \delta A \widetilde{X}_{\tilde{j}}\right\|_{F}+\phi_{2}\left\|\widehat{F}_{n_{j}} X^{-1} B^{-1} \delta B \widetilde{X}_{\tilde{j}}\right\|_{F}
$$

where, noticing that $\gamma \leq \frac{5-\sqrt{17}}{4}, \widetilde{\gamma} \leq \frac{5-\sqrt{17}}{4}$ yield $\frac{1}{1-\gamma} \frac{1+\widetilde{\gamma}}{1-\widetilde{\gamma}} \leq 2$,

$$
\phi_{1}=\left(\begin{array}{c}
\widetilde{n}_{\tilde{j}}+n_{j}-1 \\
n_{j}
\end{array}\right) \gamma^{1-n_{j}} \frac{1-\gamma^{n_{j}}}{1-\gamma} \widetilde{\gamma}^{-\widetilde{n}_{\tilde{j}}} \frac{1+\widetilde{\gamma}-2 \widetilde{\gamma}^{\widetilde{n}_{\tilde{j}}}}{1-\widetilde{\gamma}} \leq 2\left(\begin{array}{c}
\widetilde{n}_{\tilde{j}}+n_{j}-1 \\
n_{j}
\end{array}\right) \gamma^{1-n_{j}} \widetilde{\gamma}^{-\widetilde{n}_{\tilde{j}}}
$$

and similarly $\phi_{2} \leq 2\left(\begin{array}{c}\widetilde{n}_{\widetilde{j}}+n_{j}-1 \\ \widetilde{n}_{\tilde{j}}\end{array}\right) \widetilde{\gamma}^{1-\widetilde{n}_{\tilde{j}}} \gamma^{-n_{j}}$. Thus,

$$
\left\|X_{j}^{H} B \widetilde{X}_{\tilde{j}}\right\|_{F} \leq 2 \widetilde{\gamma}^{-\widetilde{n}_{\tilde{j}}} \gamma^{-n_{j}}\left\|X^{-1}\right\|_{2}\|\widetilde{X}\|_{2}\left[\gamma\left(\begin{array}{c}
\widetilde{n}_{\tilde{j}}+n_{j}-1 \\
n_{j}
\end{array}\right)\left\|A^{+} \delta A\right\|_{F}+\widetilde{\gamma}\left(\underset{\widetilde{n}_{\tilde{j}}+n_{j}-1}{\widetilde{n}_{\tilde{j}}}\right)\left\|B^{-1} \delta B\right\|_{F}\right],
$$


implying item 1.

If $n_{j}=\widetilde{n}_{\tilde{j}}=1$, then left-multiplying by $e_{\sum_{i=1}^{H} n_{i}}^{H}$ and right-multiplying by $e_{\sum_{\tilde{i}=1}^{\tilde{j}} \widetilde{n}_{\widetilde{i}}}$ (or $e_{\sum_{\tilde{i}=1}^{\tilde{j}} \widetilde{n}_{\tilde{i}}-1}$ to ensure that $\lambda, \widetilde{\lambda}$ both lie in the upper half plane), and omitting subscripts of $e_{*}$, we find that the last equation yields

$$
(\lambda-\widetilde{\lambda}) x^{H} B \widetilde{x}=-\lambda e^{H} X^{-1} A^{+} \delta A \widetilde{x} .
$$

Then it follows that

$$
|\lambda-\widetilde{\lambda}| \leq \frac{|\lambda|\left|e^{H} X^{-1} A^{+} \delta A \widetilde{x}\right|+|\widetilde{\lambda}|\left|e^{H} X^{-1} B^{-1} \delta B \widetilde{x}\right|}{\left|x^{H} B \widetilde{x}\right|} .
$$

By easy calculation, assuming that $\frac{\left|e^{H} X^{-1} B^{-1} \delta B \widetilde{x}\right|}{\left|x^{H} B \widetilde{x}\right|}<1$, this bound can be written as

$$
\frac{|\lambda-\widetilde{\lambda}|}{|\lambda|} \leq \frac{\frac{\left|e^{H} X^{-1} A^{+} \delta A \widetilde{x}\right|+\left|e^{H} X^{-1} B^{-1} \delta B \widetilde{x}\right|}{\left|x^{H} B \widetilde{x}\right|}}{1-\frac{\left|e^{H} X^{-1} B^{-1} \delta B \widetilde{x}\right|}{\left|x^{H} B \widetilde{x}\right|}} .
$$

Since

$$
\begin{aligned}
\frac{\left|e^{H} X^{-1} A^{+} \delta A \widetilde{x}\right|}{\left|x^{H} B \widetilde{x}\right|} \leq \frac{\left\|X^{-1}\right\|_{2}\|\widetilde{X}\|_{2}\left\|A^{+} \delta A\right\|_{u i}}{\left|x^{H} B \widetilde{x}\right|}, \\
\frac{\left|e^{H} X^{-1} B^{-1} \delta B \widetilde{x}\right|}{\left|x^{H} B \widetilde{x}\right|} \leq \frac{\left\|X^{-1}\right\|_{2}\|\widetilde{X}\|_{2}\left\|B^{-1} \delta B\right\|_{u i}}{\left|x^{H} B \widetilde{x}\right|},
\end{aligned}
$$

item 2 holds.

Remark 3.3. As we can see, the bounds in Theorem 3.3 are only relied on the corresponding blocks. Thus if different blocks share the same eigenvalue, then we may have a class of different bounds, in which we can choose the sharpest one.

\section{Relative perturbation bound for a regular Hermitian QEP}

In this section, we will derive perturbation bounds for eigenvectors and eigenvalues for the QEP, using results in the previous section. by

Given $M, C, K \in \mathbb{C}^{n \times n}$, the corresponding quadratic matrix polynomial of order $n$ is defined

$$
Q(\lambda)=\lambda^{2} M+\lambda C+K .
$$

$Q(\lambda)$ is called regular if $\operatorname{det} Q(\lambda)$ is not identically zero for $\lambda \in \mathbb{C}$ and singular otherwise. In this section we assume that $Q(\lambda)$ is regular. The QEP for $Q(\cdot)$ is to find $\lambda \in \mathbb{C}$ and nonzero $x \in \mathbb{C}$ such that

$$
Q(\lambda) x=\left(\lambda^{2} M+\lambda C+K\right) x=0
$$


When this equation is satisfied, $\lambda$ and $x$ are called an eigenvalue and an eigenvector, respectively. We consider the QEP (4.2), where $M$ and $K$ are Hermitian nonsingular, and $C$ is Hermitian. Instead of the QEP (4.2), similarly as in [29], we will consider the equivalent generalized eigenvalue problem

$$
\mathscr{L}_{Q}(\lambda) y=0, \quad y=\left[\begin{array}{c}
x \\
\lambda x
\end{array}\right] \in \mathbb{C}^{2 n},
$$

where $\mathscr{L}_{Q}(\lambda):=A-\lambda B$ is a matrix pair. For the case when $M$ and $K$ are nonsingular, we can obtain a symmetric linearization. For more details about linearizations of QEPs, see [28]. In this section we will consider Hermitian matrix pairs $(A, B)$, where

$$
A=\left[\begin{array}{cc}
-K & 0 \\
0 & M
\end{array}\right] \text { and } B=\left[\begin{array}{cc}
C & M \\
M & 0
\end{array}\right]
$$

The corresponding perturbed QEP is

$$
\widetilde{\lambda^{2}} \widetilde{M} \widetilde{x}+\widetilde{\lambda} \widetilde{C} \widetilde{x}+\widetilde{K} \widetilde{x}=0
$$

where $\widetilde{M}=M+\delta M$ and $\widetilde{K}=K+\delta K$ are Hermitian nonsingular and $\widetilde{C}=C+\delta C$ is Hermitian. The corresponding perturbed pair $(\widetilde{A}, \widetilde{B})$ is such that

$$
\widetilde{A}=\left[\begin{array}{cc}
-(K+\delta K) & 0 \\
0 & M+\delta M
\end{array}\right] \text { and } \widetilde{B}=\left[\begin{array}{cc}
C+\delta C & M+\delta M \\
M+\delta M & 0
\end{array}\right]
$$

We assume that the matrix pair $(A, B)$ and also the perturbed pair $(\widetilde{A}, \widetilde{B})$ satisfy assumptions (A1)-(A4). Then the result in the next theorem follows directly from Theorem 3.1.

Theorem 4.1. Given $M, C, K \in \mathbb{C}^{n \times n}$ as in (4.2). Let $\widetilde{M}=M+\delta M, \widetilde{C}=C+\delta C$ and $\widetilde{K}=K+\delta K$ be the corresponding perturbed matrices and $X$ and $\widetilde{X}$ be the nonsingular matrices representing the associated eigenspaces of the exact and perturbed QEPs. Under the assumptions (A1)-(A4) on the linearized matrix pairs (4.3) and (4.5), we have

$$
\left\|\sin \Theta\left(\operatorname{span}\left(X_{1}\right), \operatorname{span}\left(\widetilde{X}_{1}\right)\right)\right\|_{F} \leq \kappa_{2}(X) \kappa_{2}(\widetilde{X})\left(\phi_{1}^{\max } \delta a_{F}+\phi_{2}^{\max } \delta b_{F}\right),
$$

where $\phi_{1}^{\max }, \phi_{2}^{\max }$ are the same as in (3.6), and

$$
\begin{aligned}
& \delta a_{F}=\sqrt{\left\|K^{-1} \delta K\right\|_{F}^{2}+\left\|M^{-1} \delta M\right\|_{F}^{2}}, \\
& \delta b_{F}=\sqrt{2 \cdot\left\|M^{-1} \delta M\right\|_{F}^{2}+\left\|M^{-1} \delta C-M^{-1} C M^{-1} \delta M\right\|_{F}^{2}} .
\end{aligned}
$$

Proof. Bound (4.6) follows from Theorem 3.1 simply by taking the Frobenius norm of the matrices

$$
\begin{aligned}
A^{-1} \delta A & =\left[\begin{array}{cc}
-K^{-1} \delta K & 0 \\
0 & M^{-1} \delta M
\end{array}\right] \\
B^{-1} \delta B & =\left[\begin{array}{cc}
-M^{-1} \delta M & 0 \\
M^{-1} \delta C-M^{-1} C M^{-1} \delta M & M^{-1} \delta M
\end{array}\right] .
\end{aligned}
$$


Remark 4.1. As a special case, we will consider the case when both eigenvalues $\lambda$ and $\tilde{\lambda}$ of matrix pairs (4.3) and (4.5) are semi-simple. Then the columns of the matrices $X$ and $\widetilde{X}$ are the vectors

$$
X(:, i)=\left[\begin{array}{c}
x_{i} \\
\lambda_{i} x_{i}
\end{array}\right] \quad \text { and } \quad \tilde{X}(:, i)=\left[\begin{array}{c}
\widetilde{x}_{i} \\
\widetilde{\lambda}_{i} \widetilde{x}_{i}
\end{array}\right], \quad i=1, \ldots, 2 n,
$$

respectively, where $\lambda_{i}, \widetilde{\lambda}_{i}$ are eigenvalues and $x_{i}, \widetilde{x}_{i} \in \mathbb{C}^{n \times n}$ are the corresponding eigenvectors of the QEPs (4.2) and (4.4). In this case we can derive a bound for $\left|\sin \vartheta\left(x_{i}, \widetilde{x}_{i}\right)\right|$, where $\vartheta$ is the angle between the eigenvectors $x_{i}$ and $\widetilde{x}_{i}$.

Let us assume that $x_{i}$ and $\widetilde{x}_{i}$ are normalized, i.e., $\left\|x_{i}\right\|_{2}=\left\|\widetilde{x}_{i}\right\|_{2}=1$. Then by the definition of the acute angle between two unit vectors, we have that $\cos \vartheta\left(x_{i}, \widetilde{x}_{i}\right)=\left|x_{i}^{H} \widetilde{x}_{i}\right|$ and also

$$
\begin{aligned}
\cos \vartheta(X(:, i), \widetilde{X}(:, i)) & =\frac{\left|X(:, i)^{H} \widetilde{X}(:, i)\right|}{\|X(:, i)\|_{2}\|\widetilde{X}(:, i)\|_{2}} \\
& =\frac{\left|1+\overline{\lambda_{i}} \widetilde{\lambda}_{i}\right|}{\sqrt{1+\left|\lambda_{i}\right|^{2}} \sqrt{1+\left|\widetilde{\lambda}_{i}\right|^{2}}} \cdot \cos \vartheta\left(x_{i}, \widetilde{x}_{i}\right),
\end{aligned}
$$

where $\vartheta \in\left[0, \frac{\pi}{2}\right]$ and where $X(:, i)$ and $\widetilde{X}(:, i)$ are defined in (4.7). Since $\left|\left(1+\bar{\lambda}_{i} \widetilde{\lambda}_{i}\right)\right| \leq$ $\sqrt{1+\left|\lambda_{i}\right|^{2}} \sqrt{1+\left|\widetilde{\lambda}_{i}\right|^{2}}$, it is easy to see that

$$
\cos \vartheta(X(:, i), \widetilde{X}(:, i)) \leq \cos \vartheta\left(x_{i}, \widetilde{x}_{i}\right), \quad \vartheta \in\left[0, \frac{\pi}{2}\right]
$$

This means that

$$
\left|\sin \vartheta\left(x_{i}, \widetilde{x}_{i}\right)\right| \leq|\sin \vartheta(X(:, i), \widetilde{X}(:, i))| \text { for } \vartheta \in\left[0, \frac{\pi}{2}\right],
$$

and we have the same bound (4.6) for the eigenvectors $x_{i}$ and $\widetilde{x}_{i}$.

The next theorem contains upper bound for the relative errors in the eigenvalues.

Theorem 4.2. Given $M, C, K \in \mathbb{C}^{n \times n}$ as in (4.2), let $\widetilde{M}=M+\delta M, \widetilde{C}=C+\delta C$ and $\widetilde{K}=$ $K+\delta K$ be corresponding perturbed matrices and $(A, B)$ and $(\widetilde{A}, \widetilde{B})$ be the associated linearized Hermitian matrix pairs, respectively. Suppose that $A, B, \widetilde{A}, \widetilde{B}, X, \widetilde{X}$ satisfy the assumptions in Theorem 3.3. Let $\lambda$ and $\widetilde{\lambda}$ be the eigenvalues of the two matrix pairs, respectively, and $X_{\lambda}$ and $\widetilde{X}_{\widetilde{\lambda}}$ be corresponding subspaces spanned by $n_{j}, \widetilde{n}_{\tilde{j}}$ vectors, respectively. Suppose that $\lambda, \widetilde{\widetilde{\lambda}}$ are nonzero and both lie in the upper half plane, and write $\gamma=\left|\frac{\widetilde{\lambda}-\lambda}{\lambda}\right|, \widetilde{\gamma}=\left|\frac{\widetilde{\lambda}-\lambda}{\tilde{\lambda}}\right|$. Then:

1. provided $n_{j}=\widetilde{n}_{\widetilde{j}}=1$, and rewriting the eigenvectors as $X_{\lambda}=\left[\begin{array}{c}x \\ \lambda x\end{array}\right], \widetilde{X}_{\widetilde{\lambda}}=\left[\begin{array}{c}\widetilde{x} \\ \widetilde{\lambda} \widetilde{x}\end{array}\right]$, where $x$ and $\widetilde{x}$ are eigenvectors of the QEP (4.3) and (4.5), respectively, it holds

$$
\gamma \leq \frac{\left\|X^{-1}\right\|_{2}\|\widetilde{X}\|_{2}(\delta a+\delta b+\delta c)}{\delta d-\left\|X^{-1}\right\|_{2}\|\widetilde{X}\|_{2}(\delta b+\delta c)},
$$


where

$$
\begin{aligned}
\delta a & =\max \left\{\left\|K^{-1} \delta K\right\|_{2},\left\|M^{-1} \delta M\right\|_{2}\right\}, \\
\delta b & =\left\|M^{-1} \delta M\right\|_{2}, \\
\delta c & =\left\|M^{-1} \delta C\right\|_{2}+\left\|M^{-1} C M^{-1} \delta M\right\|_{2}, \\
\delta d & =\left|x^{H} C \widetilde{x}+(\widetilde{\lambda}+\bar{\lambda}) x^{H} M \widetilde{x}\right| .
\end{aligned}
$$

2. Provided $\gamma \leq \frac{5-\sqrt{17}}{4}, \widetilde{\gamma} \leq \frac{5-\sqrt{17}}{4}$, it holds

$$
\frac{\gamma^{n_{j}} \widetilde{\gamma}^{\widetilde{n}_{\tilde{j}}}}{\sqrt{\gamma^{2}+\widetilde{\gamma}^{2}}} \leq 2 \frac{\left\|X^{-1}\right\|_{2}\|\widetilde{X}\|_{2}}{\left\|X_{\lambda}^{H} B \widetilde{X}_{\tilde{\lambda}}\right\|_{F}} \sqrt{\left(\begin{array}{c}
\widetilde{n}_{\tilde{j}}+n_{j}-1 \\
n_{j}
\end{array}\right)^{2} \delta a_{F}^{2}+\left(\begin{array}{c}
\widetilde{n}_{\tilde{j}}+n_{j}-1 \\
\widetilde{n}_{\tilde{j}}
\end{array}\right)^{2} \delta b_{F}^{2}},
$$

where $\delta a_{F}$ and $\delta b_{F}$ are defined in Theorem 4.1.

Proof. Bounds (4.9) and (4.10) hold simply by Theorem 3.3, using the facts that in item 1:

$$
\begin{gathered}
\left\|A^{-1} \delta A\right\|_{2} \leq \delta a, \quad\left\|B^{-1} \delta B\right\|_{2} \leq \delta b+\delta c, \\
\left|\left[\begin{array}{cc}
x^{H} & \bar{\lambda} x^{H}
\end{array}\right] B\left[\begin{array}{c}
\widetilde{x} \\
\widetilde{\lambda} \widetilde{x}
\end{array}\right]\right|=\left|x^{H} C \widetilde{x}+\widetilde{\lambda} x^{H} M \widetilde{x}+\bar{\lambda} x^{H} M \widetilde{x}\right|,
\end{gathered}
$$

while in item 2 :

$$
\left\|A^{-1} \delta A\right\|_{F}=\delta a_{F} \quad \text { and } \quad\left\|B^{-1} \delta B\right\|_{F}=\delta b_{F} .
$$

\section{$5 \quad$ Numerical examples}

In this section, the perturbation bounds for regular Hermitian QEPs given in Section 4 will be illustrated by several numerical examples. Compared to the bound for hyperbolic QEPs given in [29], which can be considered as a special case here, the new bound is not restricted to overdamped systems, and can be applied to different mechanical systems described by nonsingular Hermitian mass $M$, nonsingular Hermitian stiffness $K$, and Hermitian damping $C$.

The numerical examples below can be grouped into two categories. If possible, we will compare the new bound with the bound given in [29, Theorem 7]. Instead of the matrix pair $A-\lambda B$ given in (4.3), the old bound is based on the equivalent matrix pair $A_{0}-\frac{1}{\lambda} J$ where

$$
A_{0}=\left[\begin{array}{cc}
L_{K}^{-1} C L_{K}^{-H} & L_{K}^{-1} L_{M} \\
L_{M}^{H} L_{K}^{-H} & 0
\end{array}\right], \quad J=\left[\begin{array}{cc}
-I & 0 \\
0 & I
\end{array}\right]
$$

and the corresponding perturbations

$$
\delta A=\left[\begin{array}{cc}
L_{K}^{-1} \delta C L_{K}^{-H} & L_{K}^{-1} \delta M L_{M}^{-H} \\
L_{M}^{-1} \delta M L_{K}^{-H} & 0
\end{array}\right], \quad \delta J=\left[\begin{array}{cc}
L_{K}^{-1} \delta K L_{K}^{-H} & 0 \\
0 & L_{M}^{-1} \delta M L_{M}^{-H}
\end{array}\right] .
$$


Here, $L_{M}$ and $L_{K}$ are the Cholesky factors of $M$ and $K$, respectively, or equivalently $M=L_{M} L_{M}^{H}$ and $K=L_{K} L_{K}^{H}$. Then the old bound is given by

$$
|\sin \vartheta(X(:, i), \widetilde{X}(:, i))| \leq \kappa_{2}(X) \kappa_{2}(\widetilde{X})\left(\frac{\left\|A_{0}^{-1} \delta A_{0}\right\|_{F}}{\min _{j \neq i} \frac{\left|\lambda_{i}-\widetilde{\lambda}_{j}\right|}{\left|\widetilde{\lambda}_{j}\right|}}+\frac{\|J \delta J\|_{F}}{\min _{j \neq i} \frac{\left|\lambda_{i}-\widetilde{\lambda}_{j}\right|}{\left|\lambda_{i}\right|}}\right),
$$

where $i=1, \ldots, 2 n$.

Otherwise, if the bound given in [29] is not valid, we will illustrate that the new bound (4.6) is applicable for the many cases when the bound (5.1) is not.

Example 5.1. This is the problem Wiresaw1 in the collection NLEVP [4]. It is a gyroscopic QEP arising in the vibration analysis of a wiresaw. More details can be found in [31]. It takes the form $G(\lambda) x=\left(\lambda^{2} M+\lambda C+K\right) x=0$, where the coefficient matrices are defined by

$$
\begin{gathered}
M=\frac{1}{2} I_{n}, \quad K=\frac{\pi^{2}\left(1-\nu^{2}\right)}{2} \operatorname{diag}\left(j^{2}\right)_{j=1, \ldots, n}, \\
C=-C^{T}=\left[c_{j k}\right]_{j, k=1, \ldots, n}, \quad \text { with } \quad c_{j k}= \begin{cases}\frac{4 j k}{j^{2}-k^{2}} \nu, & \text { if } j+k \text { is odd }, \\
0, & \text { otherwise. }\end{cases}
\end{gathered}
$$

Here, $n$ is the size of the problem and $\nu$ is a real nonnegative parameter corresponding to the speed of the wire. Clearly $M \succ 0, K$ is definite Hermitian when $\nu \neq 1$, and $C$ is skew-Hermitian. Then the quadratic matrix polynomial

$$
Q(\lambda):=-G(-\mathrm{i} \lambda)=\lambda^{2} M+\lambda(\mathrm{i} C)-K
$$

is regular and Hermitian, which implies that our bound (4.6) can be applied.

Note that for $0<\nu<1, K \succ 0$ and (5.2) is hyperbolic (but not overdamped). Then the corresponding linearization $A-\lambda B$ is positive definite and bound (5.1) for hyperbolic QEPs can also be applied. On the contrary, for $\nu>1,(5.2)$ is not hyperbolic, and then bound (5.1) is not valid.

First we choose $n=5, \nu=0.9<1$, and a group of random perturbations $\delta M, \delta C$ and $\delta K$ satisfying

$$
\left|(\delta M)_{i j}\right| \leq \eta\left|M_{i j}\right|, \quad\left|(\delta C)_{i j}\right| \leq \eta\left|C_{i j}\right|, \quad\left|(\delta K)_{i j}\right| \leq \eta\left|K_{i j}\right|,
$$

where $\eta=10^{-8}$ and matrices $M+\delta M, \mathrm{i}(C+\delta C), K+\delta K$ are also Hermitian. Then we will compare our bound (4.6) and bound (5.1) for the eigenvectors $x_{1}, \widetilde{x}_{1}$ which correspond to the eigenvalue $\lambda_{1}=21.9063$ of (5.2). The new bound (4.6) yields

$$
\left|\sin \vartheta\left(x_{1}, \widetilde{x}_{1}\right)\right| \leq 4.9283 \cdot 10^{-5},
$$

and it is not much worse than the bound (5.1) which gives

$$
\left|\sin \vartheta\left(x_{1}, \widetilde{x}_{1}\right)\right| \leq 1.1908 \cdot 10^{-6} \text {. }
$$


Then we choose $n$ and $\delta M, \delta C, \delta K$ in the same way, but $\nu=1.0019>1$. For the eigenvectors $x_{1}, \widetilde{x}_{1}$ that correspond to the eigenvalue $\lambda_{1}=-22.7864$, bound (4.6) yields

$$
\left|\sin \vartheta\left(x_{1}, \widetilde{x}_{1}\right)\right| \leq 8.5756 \cdot 10^{-6},
$$

while bound (5.1) cannot be applied. In comparison, the exact value is

$$
\left|\sin \vartheta\left(x_{1}, \widetilde{x}_{1}\right)\right| \approx 1.7615 \cdot 10^{-9} .
$$

Example 5.2. This example is related to the analysis of the behavior of brake systems and is taken from [1]. Brake squeal is a major problem in the automotive industry and it is based on the loss of stability of the brake system. In this example, we will consider negative-friction damping excitation mechanisms. Instability of the system is caused by a damping matrix $C \prec 0$.

In [1], the authors consider mechanical models of dimension 1 or 2. Here this problem is generalized to a QEP of size $n$

$$
\left(\lambda^{2} M+\lambda C+K\right) x=0,
$$

where $M, C$ and $K$ are mass, damping and stiffness matrices, respectively, and defined by

$$
M=\operatorname{diag}(j)_{j=1, \ldots, n}, \quad C=-\gamma I_{n}, \quad K=\left[\begin{array}{cccc}
10 & -5 & & \\
-5 & \ddots & \ddots & \\
& \ddots & \ddots & -5 \\
& & -5 & 10
\end{array}\right]_{n \times n} .
$$

Clearly, $M \succ 0, K \succ 0, C \prec 0$.

Here, we choose $n=4, \gamma=0.1$ and random perturbations $\delta M, \delta C$ and $\delta K$ as in (5.3). Also, we assume that the perturbed matrices satisfy $\widetilde{M}=M+\delta M \succ 0, \widetilde{K}=K+\delta K \succ 0, \widetilde{C}=C+\delta C \prec 0$.

Contrary to the bounds in [29] for hyperbolic QEPs whose eigenvalues are real, the new bounds can be applied to the complex eigenvalues and their corresponding eigenvectors. This example is designed to illustrate the sensitivity of complex eigenvalues and corresponding eigenvectors for QEPs with negative damping.

Part 1: For the eigenvectors $x_{1}, \widetilde{x}_{1}$ which correspond to the eigenvalue $\lambda_{1}=0.0251+1.1701 \mathrm{i}$, bound (4.6) gives

$$
\left|\sin \vartheta\left(x_{1}, \widetilde{x}_{1}\right)\right| \leq 6.9547 \cdot 10^{-6},
$$

in comparison with the exact value

$$
\left|\sin \vartheta\left(x_{1}, \widetilde{x}_{1}\right)\right| \approx 1.2738 \cdot 10^{-8} .
$$

Part 2: In this part, we will illustrate the performance of our eigenvalue bound (4.9). In comparison to the bounds for eigenvalues given in [30] and [29], which hold only for overdamped QEPs, our new bound is applicable to any regular Hermitian QEP. Table 5.1 shows our bound and the exact relative error for all eigenvalues $\lambda_{i} \in \Lambda(Q)$, which appear in complex conjugate pairs. 


\begin{tabular}{c|c|c}
$\lambda_{i}$ & exact value & estimate $(4.9)$ \\
\hline $0.0438 \pm 3.4550 \mathrm{i}$ & $4.8239 \mathrm{e}-09$ & $6.9836 \mathrm{e}-07$ \\
$0.0224 \pm 2.3223 \mathrm{i}$ & $6.0146 \mathrm{e}-10$ & $3.3994 \mathrm{e}-07$ \\
$0.0197 \pm 1.6640 \mathrm{i}$ & $7.8488 \mathrm{e}-10$ & $2.5006 \mathrm{e}-07$ \\
$0.0183 \pm 0.8543 \mathrm{i}$ & $3.0628 \mathrm{e}-09$ & $3.3075 \mathrm{e}-07$
\end{tabular}

Table 5.1: Example 5.2: relative perturbation bound (4.9) and exact relative error for eigenvalues.

Example 5.3. This example is constructed to show that our bound for eigenvalues and eigenvectors is also sensitive to perturbations of Jordan blocks if they appear in the canonical form of the matrix pair $(A, B)$. In this small academic example, $M, C$ and $K$ are chosen as:

$$
M=\left[\begin{array}{ll}
1 & 0 \\
0 & 2
\end{array}\right], \quad K=\left[\begin{array}{ll}
2 & 0 \\
0 & 2
\end{array}\right] \quad \text { and } \quad C=2 \cdot K .
$$

The Jordan form of the linearized pair $(A, B)$ is

$$
J=\operatorname{diag}\left\{-3.4142,-0.5858,\left[\begin{array}{cc}
-1 & 1 \\
0 & -1
\end{array}\right]\right\}
$$

Under small perturbations $\delta M, \delta C$ and $\delta K$ as in (5.3), where $\mu=10^{-7}$, we lose the size-two Jordan block of the matrix pair $(A, B)$ and all the eigenvalues of the perturbed matrix pair $(\widetilde{A}, \widetilde{B})$ are semi-simple. Table 5.2 shows that our bound in comparison to the exact value of the relative error in eigenvalues is good enough to detect the case that the structure of Jordan blocks is changed. The bounds given in [30] and [29] cannot be applied here.

\begin{tabular}{c||c|c}
$\lambda_{i}$ & exact value & estimate $(4.9)$ \\
\hline \hline-3.4142 & $2.2188 \mathrm{e}-07$ & $2.7405 \mathrm{e}-05$ \\
-0.5858 & $3.8069 \mathrm{e}-08$ & $8.0671 \mathrm{e}-07$ \\
\hline \hline$\lambda_{i}$ & exact value & estimate $(4.10)$ \\
\hline-1 & $5.6518 \mathrm{e}-08$ & $5.2899 \mathrm{e}-06$
\end{tabular}

Table 5.2: Example 5.3: relative perturbation bounds (4.9) and (4.10) and exact relative errors for eigenvalues.

The eigenvector perturbation bound (4.6) cannot directly for the eigenvectors of the QEP, so we will measure the distance between the subspaces. The subspace that we will consider is spanned by the columns of the matrices $X_{1}=X(:, 1: 2)$ of which one is the eigenvector and the other is the generalized eigenvector for the eigenvalue $\lambda_{1,2}=-1$. The perturbed subspace is spanned by the columns of the matrix $\widetilde{X}_{1}=\widetilde{X}(:, 1: 2)$ which are the corresponding eigenvectors for two distinct eigenvalues. Our bound (4.6) gives

$$
\left\|\sin \Theta\left(\operatorname{span}\left(X_{1}\right), \operatorname{span}\left(\widetilde{X}_{1}\right)\right)\right\|_{F} \leq 7.9620 \cdot 10^{-2},
$$


since $\kappa_{2}(X) \approx 3.4558, \kappa_{2}(\widetilde{X}) \approx 1.2357 \cdot 10^{4}, \phi_{1}^{\max } \approx 2.4142$ and $\phi_{2}^{\max } \approx 2.4143$. The exact distance is

$$
\left\|\sin \Theta\left(\operatorname{span}\left(X_{1}\right), \operatorname{span}\left(\widetilde{X}_{1}\right)\right)\right\|_{F} \approx 8.0927 \cdot 10^{-5},
$$

which confirms the fact that Jordan blocks are very sensitive to small perturbations and small perturbations can significantly change the corresponding invariant subspace.

\section{Conclusions}

The main contributions of this paper are new relative perturbation bounds for the eigenvalues (and their corresponding invariant subspaces) of regular Hermitian quadratic eigenvalue problems, based on the new corresponding bounds for regular Hermitian pairs. The obtained bounds can be applied to many interesting problems, for example, to quadratic eigenvalue problems appearing in many mechanical models, especially models with indefinite damping or mass matrices. The main advantage of the new bounds, compared to earlier bounds, is that they are more general and can be applied not only to hyperbolic quadratic eigenvalue problems, but also to other regular quadratic eigenvalue problems. The quality of our bounds has been illustrated using several numerical examples.

\section{A Proof of Lemma 3.1}

In the very beginning, we point out our way to prove the lemma. The key is to vectorize the equations (3.1a) and (3.1b). Then we use standard but complicated calculation to estimate the upper bound of the solution.

Consider (3.1a). Recall that $(\Lambda, \Omega)$ and $\left(\Lambda^{\prime}, \Omega^{\prime}\right)$ are of type R2, R3 or R4. Then $\Omega^{\prime} \Lambda^{\prime}$ and $\Omega \Lambda$ are lower triangular. Thus, $\left(I \otimes \Omega^{\prime} \Lambda^{\prime}-\Omega \Lambda \otimes I\right)$ is a lower triangular matrix. Each diagonal entry is $\lambda-\lambda^{\prime}$, so this matrix is nonsingular. We have

$$
\begin{aligned}
\operatorname{vec}(Y) & =\left(I \otimes \Omega^{\prime} \Lambda^{\prime}-\Omega \Lambda \otimes I\right)^{-H}\left[-\left(I \otimes \Omega^{\prime} \Lambda^{\prime}\right)^{H} \operatorname{vec}(M)+(\Omega \Lambda \otimes I)^{H} \operatorname{vec}(N)\right] \\
& =:-W_{1}^{H} \operatorname{vec}(M)+W_{2}^{H} \operatorname{vec}(N),
\end{aligned}
$$

where $W_{1}=\left(I \otimes \Omega^{\prime} \Lambda^{\prime}\right)\left(I \otimes \Omega^{\prime} \Lambda^{\prime}-\Omega \Lambda \otimes I\right)^{-1}, W_{2}=(\Omega \Lambda \otimes I)\left(I \otimes \Omega^{\prime} \Lambda^{\prime}-\Omega \Lambda \otimes I\right)^{-1}$. This shows (3.1a) has a unique solution $Y$, and $W_{1}-W_{2}=I$. Moreover,

$$
\|Y\|_{F} \leq\left\|W_{1}\right\|_{2}\|M\|_{F}+\left\|W_{2}\right\|_{2}\|N\|_{F} .
$$

Compared to the result, the rest of the proof is to estimate $\left\|W_{1}\right\|_{2}$ and $\left\|W_{2}\right\|_{2}$.

Considering the types of $(\Lambda, \Omega)$ and $\left(\Lambda^{\prime}, \Omega^{\prime}\right)$, there are eight cases to discuss. Before that, first we explicitly invert a structured matrix, which will be used several times in the following. Consider the block matrix $P=D_{1} \otimes L+F D_{2} G \otimes I=\left[P_{i, j}\right]_{i, j=1, \ldots, m}$ with $D_{1}=$ $\operatorname{diag}\left(d_{1,1}, \ldots, d_{1, m}\right), D_{2}=\operatorname{diag}\left(d_{2,1}, \ldots, d_{2, m}\right)$ and $L \in \mathbb{C}^{n \times n}$ lower triangular. Specifically,

$$
P_{i, j}= \begin{cases}0, & i<j \text { or } i>j+1, \\ d_{1, j} L, & i=j, \\ d_{2, m-j} I, & i=j+1 .\end{cases}
$$


Assume that $D_{1}$ and $L$ are nonsingular, and hence $P_{i, j}$ and also $P$ are nonsingular. Then its inverse $P^{-1}=\left[Q_{i, j}\right]_{i, j=1, \ldots, m}$ satisfies

$$
Q_{i, j}= \begin{cases}\prod_{k=1}^{i-j}\left(-P_{i-k+1, i-k+1}^{-1} P_{i-k+1, i-k}\right) Q_{j, j}, & i>j, \\ 0, & i<j .\end{cases}
$$

Since $P$ is block lower triangular, $Q_{j, j}=d_{1, j}^{-1} L^{-1}$. Calculating recursively, we have ${ }^{1}$

$$
Q= \begin{cases}(-1)^{i-j} \prod_{k=1}^{i-j+1} d_{1, i-k+1}^{-1} \prod_{k=1}^{i-j} d_{2, m-i+k} L^{j-i-1}, & i \geq j \\ 0, & i<j .\end{cases}
$$

Now we can go back for the eight cases.

1. $(\Lambda, \Omega)$ and $\left(\Lambda^{\prime}, \Omega^{\prime}\right)$ are both of type $\mathbf{R 3 :}$

$$
\begin{aligned}
I \otimes \Omega^{\prime} \Lambda^{\prime}-\Omega \Lambda \otimes I & =I \otimes\left(\lambda^{\prime} I+\left|\lambda^{\prime}\right| F G\right)-(\lambda I+|\lambda| F G) \otimes I \\
& =I \otimes\left(\left[\lambda^{\prime}-\lambda\right] I+\left|\lambda^{\prime}\right| F G\right)-|\lambda| F G \otimes I,
\end{aligned}
$$

and then by (A.1)

$$
\left(I \otimes \Omega^{\prime} \Lambda^{\prime}-\Omega \Lambda \otimes I\right)^{-1}= \begin{cases}|\lambda|^{i-j}\left(\left[\lambda^{\prime}-\lambda\right] I+\left|\lambda^{\prime}\right| F G\right)^{j-i-1}, & i \geq j, \\ 0, & i<j,\end{cases}
$$

where also by (A.1)

$$
\left(\left[\lambda^{\prime}-\lambda\right] I+\left|\lambda^{\prime}\right| F G\right)^{-1}= \begin{cases}\left(-\left|\lambda^{\prime}\right|\right)^{i^{\prime}-j^{\prime}}\left(\lambda^{\prime}-\lambda\right)^{j^{\prime}-i^{\prime}-1}, & i^{\prime} \geq j^{\prime}, \\ 0, & i^{\prime}<j^{\prime}\end{cases}
$$

Then

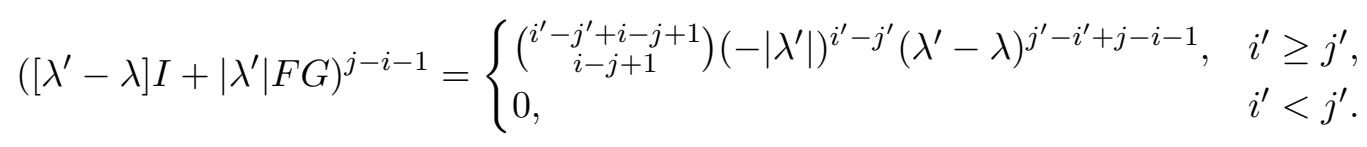

Thus

$$
W_{1}= \begin{cases}|\lambda|^{i-j}\left(\lambda^{\prime} I+\left|\lambda^{\prime}\right| F G\right)\left(\left[\lambda^{\prime}-\lambda\right] I+\left|\lambda^{\prime}\right| F G\right)^{j-i-1}, & i \geq j \\ 0, & i<j\end{cases}
$$

where

$$
\begin{aligned}
\left(\lambda^{\prime} I\right. & \left.+\left|\lambda^{\prime}\right| F G\right)\left(\left[\lambda^{\prime}-\lambda\right] I+\left|\lambda^{\prime}\right| F G\right)^{j-i-1} \\
& = \begin{cases}\left.\left(-\left|\lambda^{\prime}\right|\right)^{i^{\prime}-j^{\prime}}\left(\lambda^{\prime}-\lambda\right)^{j^{\prime}-i^{\prime}+j-i}\left[\begin{array}{c}
i^{\prime}-j^{\prime}+i-j+1 \\
i-j+1
\end{array}\right) \lambda^{\prime}\left(\lambda^{\prime}-\lambda\right)^{-1}-\left(\begin{array}{c}
i^{\prime}-j^{\prime}+i-j \\
i-j+1
\end{array}\right)\right], & i^{\prime} \geq j^{\prime}, \\
0, & i^{\prime}<j^{\prime} .\end{cases}
\end{aligned}
$$

\footnotetext{
${ }^{1}$ Actually the left-hand side is $Q_{i, j}$, but we abuse the notation $Q$ to denote the matrix.
} 
It is easy to see $\left\|W_{1}\right\|_{1}=\left\|W_{1}\right\|_{\infty}=\left\|W_{1} e_{1}\right\|_{1}$. Note that $\left\|W_{1}\right\|_{2} \leq \sqrt{\left\|W_{1}\right\|_{1}\left\|W_{1}\right\|_{\infty}}=$ $\left\|W_{1}\right\|_{1}$. Thus,

$$
\begin{aligned}
& \left\|W_{1}\right\|_{2} \leq\left\|W_{1} e_{1}\right\|_{1} \\
& =\left.\sum_{i=1}^{n} \sum_{i^{\prime}=1}^{n^{\prime}}|| \lambda\right|^{i-1}\left(-\left|\lambda^{\prime}\right|\right)^{i^{\prime}-1}\left(\lambda^{\prime}-\lambda\right)^{2-i^{\prime}-i}\left[\left(\begin{array}{c}
i^{\prime}+i-1 \\
i
\end{array}\right) \lambda^{\prime}\left(\lambda^{\prime}-\lambda\right)^{-1}-\left(\begin{array}{c}
i^{\prime}+i-2 \\
i
\end{array}\right)\right] \mid \\
& \leq \sum_{i=1}^{n} \sum_{i^{\prime}=1}^{n^{\prime}} \gamma^{1-i} \gamma^{\prime 1-i^{\prime}}\left[\left(\begin{array}{c}
i^{\prime}+i-1 \\
i
\end{array}\right) \gamma^{\prime-1}+\left(\begin{array}{c}
i^{\prime}+i-2 \\
i
\end{array}\right)\right] \\
& =2 \sum_{i=1}^{n} \sum_{i^{\prime}=1}^{n^{\prime}-1} \gamma^{1-i} \gamma^{\prime-i^{\prime}}\left(\begin{array}{c}
i^{\prime}+i-1 \\
i
\end{array}\right)+\sum_{i=1}^{n} \gamma^{1-i} \gamma^{\prime-n^{\prime}}\left(\begin{array}{c}
n^{\prime}+i-1 \\
i
\end{array}\right) \\
& \leq\left(2 \sum_{i=1}^{n} \sum_{i^{\prime}=1}^{n^{\prime}-1} \gamma^{1-i} \gamma^{\prime-i^{\prime}}+\sum_{i=1}^{n} \gamma^{1-i} \gamma^{\prime-n^{\prime}}\right)\left(\begin{array}{c}
n^{\prime}+n-1 \\
n
\end{array}\right) \\
& =\left(2 \gamma^{\prime-1} \frac{1-\gamma^{\prime 1-n^{\prime}}}{1-\gamma^{\prime-1}} \frac{1-\gamma^{-n}}{1-\gamma^{-1}}+\gamma^{\prime-n^{\prime}} \frac{1-\gamma^{-n}}{1-\gamma^{-1}}\right)\left(\begin{array}{c}
n^{\prime}+n-1 \\
n
\end{array}\right) \\
& =\gamma^{\prime-1} \frac{2-\gamma^{1-n^{\prime}}-\gamma^{\prime-n^{\prime}}}{1-\gamma^{\prime-1}} \frac{1-\gamma^{-n}}{1-\gamma^{-1}}\left(\begin{array}{c}
n^{\prime}+n-1 \\
n
\end{array}\right) \\
& =\gamma^{\prime-n^{\prime}} \gamma^{1-n} \frac{1+\gamma^{\prime}-2 \gamma^{\prime n^{\prime}}}{1-\gamma^{\prime}} \frac{1-\gamma^{n}}{1-\gamma}\left(\begin{array}{c}
n^{\prime}+n-1 \\
n
\end{array}\right) \\
& =\left(\begin{array}{c}
n^{\prime}+n-1 \\
n
\end{array}\right) \varphi_{-}(\gamma, n) \varphi_{+}\left(\gamma^{\prime}, n^{\prime}\right) \text {. }
\end{aligned}
$$

Similarly,

$$
\left\|W_{2}\right\|_{2} \leq\left(\begin{array}{c}
n^{\prime}+n-1 \\
n^{\prime}
\end{array}\right) \varphi_{-}\left(\gamma^{\prime}, n^{\prime}\right) \varphi_{+}(\gamma, n) .
$$

2. $(\Lambda, \Omega)$ and $\left(\Lambda^{\prime}, \Omega^{\prime}\right)$ are both of type $\mathbf{R} 4$ :

$$
\begin{aligned}
I \otimes \Omega^{\prime} \Lambda^{\prime}-\Omega \Lambda \otimes I & =\left[\begin{array}{ll}
I & \\
& I
\end{array}\right] \otimes\left[\begin{array}{ll}
\lambda^{\prime} I+\left|\lambda^{\prime}\right| F G & \overline{\lambda^{\prime}} I+\left|\overline{\lambda^{\prime}}\right| F G
\end{array}\right] \\
& -\left[\begin{array}{ll}
\lambda I+|\lambda| F G & \bar{\lambda} I+|\bar{\lambda}| F G
\end{array}\right] \otimes\left[\begin{array}{ll}
I & \\
& I
\end{array}\right] \\
& =\operatorname{diag}\left(R_{1}, R_{2}, \overline{R_{1}}, \overline{R_{2}}\right),
\end{aligned}
$$

where

$$
\begin{aligned}
& R_{1}=\left[I \otimes\left(\lambda^{\prime} I+\left|\lambda^{\prime}\right| F G\right)-(\lambda I+|\lambda| F G) \otimes I\right], \\
& R_{2}=\left[I \otimes\left(\overline{\lambda^{\prime}} I+\left|\lambda^{\prime}\right| F G\right)-(\lambda I+|\lambda| F G) \otimes I\right] .
\end{aligned}
$$

Then

$$
\begin{aligned}
W_{1}=\operatorname{diag}\left(R_{1}^{-1}\left(\lambda^{\prime} I+\left|\lambda^{\prime}\right| F G\right),\right. & R_{2}^{-1}\left(\overline{\lambda^{\prime}} I+\left|\lambda^{\prime}\right| F G\right), \\
& \left.\bar{R}_{1}^{-1}\left(\lambda^{\prime} I+\left|\lambda^{\prime}\right| F G\right),{\overline{R_{2}}}^{-1}\left(\overline{\lambda^{\prime}} I+\left|\lambda^{\prime}\right| F G\right)\right) .
\end{aligned}
$$


Write $\gamma_{1}:=\left|\frac{\lambda^{\prime}-\lambda}{\lambda}\right|, \gamma_{1}^{\prime}:=\left|\frac{\lambda^{\prime}-\lambda}{\lambda^{\prime}}\right|, \gamma_{2}:=\left|\frac{\overline{\lambda^{\prime}}-\lambda}{\lambda}\right|, \gamma_{2}^{\prime}:=\left|\frac{\overline{\lambda^{\prime}}-\lambda}{\lambda^{\prime}}\right|$, then by the same calculation in case 1 we have

$$
\begin{aligned}
& \left\|R_{1}^{-1}\left(\lambda^{\prime} I+\left|\lambda^{\prime}\right| F G\right)\right\|_{2} \leq\left(\begin{array}{c}
n^{\prime}+n-1 \\
n
\end{array}\right) \varphi_{-}\left(\gamma_{1}, n\right) \varphi_{+}\left(\gamma_{1}^{\prime}, n^{\prime}\right) \\
& \left\|R_{2}^{-1}\left(\overline{\lambda^{\prime}} I+\left|\lambda^{\prime}\right| F G\right)\right\|_{2} \leq\left(\begin{array}{c}
n^{\prime}+n-1 \\
n
\end{array}\right) \varphi_{-}\left(\gamma_{2}, n\right) \varphi_{+}\left(\gamma_{2}^{\prime}, n^{\prime}\right) .
\end{aligned}
$$

Similarly to the calculation in case 1 we get

$$
{\overline{R_{1}}}^{-1}\left(\lambda^{\prime} I+\left|\lambda^{\prime}\right| F G\right)= \begin{cases}|\lambda|^{i-j}\left(\lambda^{\prime} I+\left|\lambda^{\prime}\right| F G\right)\left(\left[\overline{\lambda^{\prime}}-\bar{\lambda}\right] I+\left|\lambda^{\prime}\right| F G\right)^{j-i-1}, & i \geq j, \\ 0, & i<j,\end{cases}
$$

where

$$
\begin{aligned}
\left(\lambda^{\prime} I\right. & \left.+\left|\lambda^{\prime}\right| F G\right)\left(\left[\overline{\lambda^{\prime}}-\bar{\lambda}\right] I+\left|\lambda^{\prime}\right| F G\right)^{j-i-1} \\
& = \begin{cases}\left.\left(-\left|\lambda^{\prime}\right|\right)^{i^{\prime}-j^{\prime}}\left(\overline{\lambda^{\prime}}-\bar{\lambda}\right)^{j^{\prime}-i^{\prime}+j-i}\left[\begin{array}{c}
i^{\prime}-j^{\prime}+i-j+1 \\
i-j+1
\end{array}\right) \lambda^{\prime}\left(\overline{\lambda^{\prime}}-\bar{\lambda}\right)^{-1}-\left(\begin{array}{c}
i^{\prime}-j^{\prime}+i-j \\
i-j+1
\end{array}\right)\right], & i^{\prime} \geq j^{\prime}, \\
0, & i^{\prime}<j^{\prime},\end{cases}
\end{aligned}
$$

and then

$$
\left\|{\overline{R_{1}}}^{-1}\left(\lambda^{\prime} I+\left|\lambda^{\prime}\right| F G\right)\right\|_{2} \leq\left(\begin{array}{c}
n^{\prime}+n-1 \\
n
\end{array}\right) \varphi_{-}\left(\gamma_{1}, n\right) \varphi_{+}\left(\gamma_{1}^{\prime}, n^{\prime}\right)
$$

similarly,

$$
\left\|{\overline{R_{2}}}^{-1}\left(\overline{\lambda^{\prime}} I+\left|\lambda^{\prime}\right| F G\right)\right\|_{2} \leq\left(\begin{array}{c}
n^{\prime}+n-1 \\
n
\end{array}\right) \varphi_{-}\left(\gamma_{2}, n\right) \varphi_{+}\left(\gamma_{2}^{\prime}, n^{\prime}\right) .
$$

In summary,

$$
\left\|W_{1}\right\|_{2} \leq\left(\begin{array}{c}
n^{\prime}+n-1 \\
n
\end{array}\right) \varphi_{-}(\gamma, n) \varphi_{+}\left(\gamma^{\prime}, n^{\prime}\right),
$$

where $\gamma=\min \left\{\gamma_{1}, \gamma_{2}\right\}$. Similarly,

$$
\left\|W_{2}\right\|_{2} \leq\left(\begin{array}{c}
n^{\prime}+n-1 \\
n^{\prime}
\end{array}\right) \varphi_{-}\left(\gamma^{\prime}, n^{\prime}\right) \varphi_{+}(\gamma, n) .
$$

3. $(\Lambda, \Omega)$ is of type $\mathbf{R 2}$, while $\left(\Lambda^{\prime}, \Omega^{\prime}\right)$ is of type $\mathbf{R 3}$ :

$$
I \otimes \Omega^{\prime} \Lambda^{\prime}-\Omega \Lambda \otimes I=I \otimes\left(\lambda^{\prime} I+\left|\lambda^{\prime}\right| F G\right)-F G \otimes I,
$$

and then

$$
\left(I \otimes \Omega^{\prime} \Lambda^{\prime}-\Omega \Lambda \otimes I\right)^{-1}= \begin{cases}\left(\lambda^{\prime} I+\left|\lambda^{\prime}\right| F G\right)^{j-i-1}, & i \geq j \\ 0, & i<j\end{cases}
$$

where

$$
\left(\lambda^{\prime} I+\left|\lambda^{\prime}\right| F G\right)^{-1}= \begin{cases}\left(-\left|\lambda^{\prime}\right|\right)^{i^{\prime}-j^{\prime}} \lambda^{\prime j^{\prime}-i^{\prime}-1}, & i^{\prime} \geq j^{\prime} \\ 0, & i^{\prime}<j^{\prime}\end{cases}
$$

Thus

$$
W_{1}= \begin{cases}\left(\lambda^{\prime} I+\left|\lambda^{\prime}\right| F G\right)^{j-i}, & i \geq j \\ 0, & i<j\end{cases}
$$


where

$$
\left(\lambda^{\prime} I+\left|\lambda^{\prime}\right| F G\right)^{j-i}=\left\{\begin{array}{ll}
\left(-\left|\lambda^{\prime}\right|\right)^{i^{\prime}-j^{\prime}} \lambda^{\prime j^{\prime}-i^{\prime}+j-i}\left(\begin{array}{c}
i^{\prime}-j^{\prime}+i-j \\
i-j
\end{array}\right), & i^{\prime} \geq j^{\prime}, \\
0, & i^{\prime}<j^{\prime},
\end{array} \quad \text { for } i>j .\right.
$$

It is easy to see $\left\|W_{1}\right\|_{1}=\left\|W_{1}\right\|_{\infty}=\left\|W_{1} e_{1}\right\|_{1}$. Note that $\left\|W_{1}\right\|_{2} \leq \sqrt{\left\|W_{1}\right\|_{1}\left\|W_{1}\right\|_{\infty}}=$ $\left\|W_{1}\right\|_{1}$. Thus,

$$
\begin{aligned}
\left\|W_{1}\right\|_{2} & \leq\left\|W_{1} e_{1}\right\|_{1} \\
& =1+\sum_{i=2}^{n} \sum_{i^{\prime}=1}^{n^{\prime}}\left|\left(-\left|\lambda^{\prime}\right|\right)^{i^{\prime}-1} \lambda^{\prime 2-i^{\prime}-i}\left(\begin{array}{c}
i^{\prime}+i-2 \\
i-1
\end{array}\right)\right| \\
& =1+\sum_{i=2}^{n} \sum_{i^{\prime}=1}^{n^{\prime}}\left|\lambda^{\prime}\right|^{1-i}\left(\begin{array}{c}
i^{\prime}+i-2 \\
i-1
\end{array}\right) .
\end{aligned}
$$

$\operatorname{Noticing}^{2} \sum_{i^{\prime}=1}^{n^{\prime}}\left(\begin{array}{c}i^{\prime}+i-2 \\ i-1\end{array}\right)=\left(\begin{array}{c}i-1 \\ i-1\end{array}\right)+\sum_{i^{\prime}=2}^{n^{\prime}}\left(\begin{array}{c}i^{\prime}+i-2 \\ i-1\end{array}\right)=\left(\begin{array}{l}i \\ i\end{array}\right)+\sum_{i^{\prime}=2}^{n^{\prime}}\left(\begin{array}{c}i^{\prime}+i-2 \\ i-1\end{array}\right)=\cdots=\left(\begin{array}{c}n^{\prime}+i-1 \\ i\end{array}\right)$,

$$
\begin{aligned}
\left\|W_{1}\right\|_{2} & \leq 1+\sum_{i=2}^{n}\left|\lambda^{\prime}\right|^{1-i}\left(\begin{array}{c}
n^{\prime}+i-1 \\
i
\end{array}\right) \\
& \leq 1+\frac{\left|\lambda^{\prime}\right|^{-1}-\left|\lambda^{\prime}\right|^{-n}}{1-\left|\lambda^{\prime}\right|^{-1}}\left(\begin{array}{c}
n^{\prime}+n-1 \\
n
\end{array}\right) \\
& =1+\left(\begin{array}{c}
n^{\prime}+n-1 \\
n
\end{array}\right)\left|\lambda^{\prime}\right|^{-1} \varphi_{-}\left(\left|\lambda^{\prime}\right|, n-1\right) .
\end{aligned}
$$

Then

$$
\begin{aligned}
\left\|W_{2}\right\|_{2} & \leq\left\|W_{1} e_{1}-e_{1}\right\|_{1} \\
& =\sum_{i=2}^{n} \sum_{i^{\prime}=1}^{n^{\prime}}\left|\left(-\left|\lambda^{\prime}\right|\right)^{i^{\prime}-1} \lambda^{\prime 2-i^{\prime}-i}\left(\begin{array}{c}
i^{\prime}+i-2 \\
i-1
\end{array}\right)\right| \\
& =\left(\begin{array}{c}
n^{\prime}+n-1 \\
n
\end{array}\right)\left|\lambda^{\prime}\right|^{-1} \varphi_{-}\left(\left|\lambda^{\prime}\right|, n-1\right) .
\end{aligned}
$$

4. $(\Lambda, \Omega)$ is of type $\mathbf{R 2}$, while $\left(\Lambda^{\prime}, \Omega^{\prime}\right)$ is of type $\mathbf{R} 4$ :

$$
\begin{aligned}
I \otimes \Omega^{\prime} \Lambda^{\prime}-\Omega \Lambda \otimes I & =I \otimes\left[\begin{array}{ll}
\lambda^{\prime} I+\left|\lambda^{\prime}\right| F G & \\
& \overline{\lambda^{\prime}} I+\left|\overline{\lambda^{\prime}}\right| F G
\end{array}\right]-F G \otimes\left[\begin{array}{ll}
I & \\
& I
\end{array}\right] \\
& =\operatorname{diag}\left(R_{1}, \overline{R_{1}}\right),
\end{aligned}
$$

where

$$
R_{1}=\left[I \otimes\left(\lambda^{\prime} I+\left|\lambda^{\prime}\right| F G\right)-F G \otimes I\right] .
$$

Then

$$
W_{1}=\operatorname{diag}\left(R_{1}^{-1}\left(\lambda^{\prime} I+\left|\lambda^{\prime}\right| F G\right),{\overline{R_{1}}}^{-1}\left(\overline{\lambda^{\prime}} I+\left|\lambda^{\prime}\right| F G\right)\right) .
$$

\footnotetext{
${ }^{2}\left(\begin{array}{c}p \\ q-1\end{array}\right)+\left(\begin{array}{c}p \\ q\end{array}\right)=\left(\begin{array}{c}p+1 \\ q\end{array}\right)$ for any $q \in \mathbb{N}_{+}$.
} 
By the same calculation in case 3 ,

$$
\left\|R_{1}^{-1}\left(\lambda^{\prime} I+\left|\lambda^{\prime}\right| F G\right)\right\|_{2} \leq\left(\begin{array}{c}
n^{\prime}+n-1 \\
n
\end{array}\right) \varphi_{-}\left(\left|\lambda^{\prime}\right|, n\right) ;
$$

Note that

$$
\left\|{\overline{R_{1}}}^{-1}\left(\overline{\lambda^{\prime}} I+\left|\lambda^{\prime}\right| F G\right)\right\|_{2}=\left\|R_{1}^{-1}\left(\lambda^{\prime} I+\left|\lambda^{\prime}\right| F G\right)\right\|_{2} .
$$

Thus

$$
\left\|W_{1}\right\|_{2} \leq\left(\begin{array}{c}
n^{\prime}+n-1 \\
n
\end{array}\right) \varphi_{-}\left(\left|\lambda^{\prime}\right|, n\right) .
$$

Similarly,

$$
\left\|W_{2}\right\|_{2} \leq\left(\begin{array}{c}
n^{\prime}+n-1 \\
n
\end{array}\right) \varphi_{-}\left(\left|\lambda^{\prime}\right|, n^{\prime}\right)-1 .
$$

5. $(\Lambda, \Omega)$ is of type $\mathbf{R 3}$, while $\left(\Lambda^{\prime}, \Omega^{\prime}\right)$ is of type $\mathbf{R} 4$ :

$$
\begin{aligned}
I \otimes \Omega^{\prime} \Lambda^{\prime}-\Omega \Lambda \otimes I & =I \otimes\left[\begin{array}{ll}
\lambda^{\prime} I+\left|\lambda^{\prime}\right| F G & \\
& \overline{\lambda^{\prime}} I+\left|\overline{\lambda^{\prime}}\right| F G
\end{array}\right]-(\lambda I+|\lambda| F G) \otimes\left[\begin{array}{ll}
I & \\
& I
\end{array}\right] \\
& =\operatorname{diag}\left(R_{1}, \overline{R_{1}}\right),
\end{aligned}
$$

where

$$
R_{1}=\left[I \otimes\left(\lambda^{\prime} I+\left|\lambda^{\prime}\right| F G\right)-(\lambda I+|\lambda| F G) \otimes I\right] .
$$

Then

$$
W_{1}=\operatorname{diag}\left(R_{1}^{-1}\left(\lambda^{\prime} I+\left|\lambda^{\prime}\right| F G\right),{\overline{R_{1}}}^{-1}\left(\overline{\lambda^{\prime}} I+\left|\lambda^{\prime}\right| F G\right)\right) .
$$

By the same calculation in case 1 ,

$$
\left\|R_{1}^{-1}\left(\lambda^{\prime} I+\left|\lambda^{\prime}\right| F G\right)\right\|_{2} \leq\left(\begin{array}{c}
n^{\prime}+n-1 \\
n
\end{array}\right) \varphi_{-}(\gamma, n) \varphi_{+}\left(\gamma^{\prime}, n^{\prime}\right)
$$

Note that

$$
\left\|{\overline{R_{1}}}^{-1}\left(\overline{\lambda^{\prime}} I+\left|\lambda^{\prime}\right| F G\right)\right\|_{2}=\left\|R_{1}^{-1}\left(\lambda^{\prime} I+\left|\lambda^{\prime}\right| F G\right)\right\|_{2} .
$$

Thus

$$
\left\|W_{1}\right\|_{2} \leq\left(\begin{array}{c}
n^{\prime}+n-1 \\
n
\end{array}\right) \varphi_{-}(\gamma, n) \varphi_{+}\left(\gamma^{\prime}, n^{\prime}\right) .
$$

Similarly,

$$
\left\|W_{2}\right\|_{2} \leq\left(\begin{array}{c}
n^{\prime}+n-1 \\
n^{\prime}
\end{array}\right) \varphi_{-}\left(\gamma^{\prime}, n^{\prime}\right) \varphi_{+}(\gamma, n) .
$$

6. $(\Lambda, \Omega)$ is of type $\mathbf{R 3}$, while $\left(\Lambda^{\prime}, \Omega^{\prime}\right)$ is of type $\mathbf{R 2}$ : Note that there exist two permutation matrices $P, Q$ such that $A \otimes B=P(B \otimes A) Q$. Thus, $\|A \otimes B\|_{2}=\|B \otimes A\|_{2}$. So it is the same as case 3 .

7. $(\Lambda, \Omega)$ is of type $\mathbf{R} 4$, while $\left(\Lambda^{\prime}, \Omega^{\prime}\right)$ is of type $\mathbf{R 2}$ : the same as case 4 .

8. $(\Lambda, \Omega)$ is of type $\mathbf{R} 4$, while $\left(\Lambda^{\prime}, \Omega^{\prime}\right)$ is of type $\mathbf{R 3}$ : the same as case 5 . 
Consider (3.1b). Recall that $(\Lambda, \Omega)$ is of type R2, R3 or R4. Then $\Omega \Lambda$ are lower triangular as well as $F G$. Thus, $(I \otimes I-\Omega \Lambda \otimes F G)$ is a lower triangular matrix. Each diagonal entry is 1 , so this matrix is nonsingular. Then

$$
\begin{aligned}
\operatorname{vec}(Y) & =(I \otimes I-\Omega \Lambda \otimes F G)^{-H}\left[-(I \otimes I)^{H} \operatorname{vec}(M)+(\Omega \Lambda \otimes F G)^{H} \operatorname{vec}(N)\right] \\
& =:-W_{1}^{H} \operatorname{vec}(M)+W_{2}^{H} \operatorname{vec}(N),
\end{aligned}
$$

where $W_{1}=(I \otimes I)(I \otimes I-\Omega \Lambda \otimes F G)^{-1}, W_{2}=(\Omega \Lambda \otimes F G)(I \otimes I-\Omega \Lambda \otimes F G)^{-1}$. Similarly to the discussion on (3.1a), (3.1b) has a unique solution $Y, W_{1}-W_{2}=I$, and the rest of the proof is to estimate $\left\|W_{1}\right\|_{2}$ and $\left\|W_{2}\right\|_{2}$.

Note that there exist two permutation matrices $P, Q$ to make $A \otimes B=P(B \otimes A) Q$. Thus, $\|A \otimes B\|_{2}=\|B \otimes A\|_{2}$. Then

$$
I \otimes I-F G \otimes \Omega \Lambda=\left[\begin{array}{cccc}
I & & & \\
\Omega \Lambda & I & & \\
& \ddots & \ddots & \\
& & \Omega \Lambda & I
\end{array}\right]
$$

and

$$
W_{0}:=(I \otimes I-F G \otimes \Omega \Lambda)^{-1}= \begin{cases}(-\Omega \Lambda)^{i^{\prime}-j^{\prime}}, & i^{\prime} \geq j^{\prime} \\ 0, & i^{\prime}<j^{\prime}\end{cases}
$$

Then

$$
\left\|W_{1}\right\|_{2}=\left\|(I \otimes I-\Omega \Lambda \otimes F G)^{-1}\right\|_{2}=\left\|(I \otimes I-F G \otimes \Omega \Lambda)^{-1}\right\|_{2}=\left\|W_{0}\right\|_{2} .
$$

There are three cases to discuss in the following, and for whatever cases it is easy to see that $\left\|W_{0}\right\|_{1}=\left\|W_{0}\right\|_{\infty}=\left\|W_{0} e_{1}\right\|_{1}$ and then $\left\|W_{0}\right\|_{2} \leq \sqrt{\left\|W_{0}\right\|_{1}\left\|W_{0}\right\|_{\infty}}=\left\|W_{0}\right\|_{1}=\left\|W_{0} e_{1}\right\|_{1}$.

1. if $(\Lambda, \Omega)$ is of $\mathbf{R 2 :}$

$$
(-\Omega \Lambda)^{i^{\prime}-j^{\prime}}= \begin{cases}(-1)^{i^{\prime}-j^{\prime}}, & i-j=i^{\prime}-j^{\prime} \\ 0, & i-j \neq i^{\prime}-j^{\prime} .\end{cases}
$$

Thus $\left\|W_{1}\right\|_{2} \leq\left\|W_{0} e_{1}\right\|_{1}=\min \left\{n, n^{\prime}\right\} ;$ and $\left\|W_{2}\right\|_{2} \leq\left\|W_{0} e_{1}-e_{1}\right\|_{1}=\min \left\{n, n^{\prime}\right\}-1$.

2. if $(\Lambda, \Omega)$ is of $\mathbf{R 3}$ :

$$
(-\Omega \Lambda)^{i^{\prime}-j^{\prime}}=\left\{\begin{array}{ll}
(-1)^{i^{\prime}-j^{\prime}} \lambda^{i^{\prime}-j^{\prime}-i+j}|\lambda|^{i-j}\left(\begin{array}{c}
i^{\prime}-j^{\prime} \\
i-j
\end{array}\right), & i \geq j, \\
0, & i<j,
\end{array} \text { for } i^{\prime} \geq j^{\prime} .\right.
$$

Thus

$$
\begin{aligned}
\left\|W_{1}\right\|_{2} & \leq\left\|W_{0} e_{1}\right\|_{1} \\
& =1+\left.\sum_{i=1}^{n} \sum_{i^{\prime}=2}^{n^{\prime}}\left|(-1)^{i^{\prime}-1} \lambda^{i^{\prime}-i}\right| \lambda\right|^{i-1}\left(\begin{array}{c}
i^{\prime}-1 \\
i-1
\end{array}\right) \mid \\
& =1+\sum_{i=1}^{n} \sum_{i^{\prime}=2}^{n^{\prime}}|\lambda|^{i^{\prime}-1}\left(\begin{array}{c}
i^{\prime}-1 \\
i-1
\end{array}\right)
\end{aligned}
$$




$$
\begin{aligned}
& \leq 1+\sum_{i^{\prime}=2}^{n^{\prime}}|\lambda|^{i^{\prime}-1} 2^{i^{\prime}-1} \\
& =1+\frac{2|\lambda|-(2|\lambda|)^{n^{\prime}}}{1-2|\lambda|} \\
& =1+2|\lambda| \varphi\left(\frac{1}{2|\lambda|}, n^{\prime}-1\right),
\end{aligned}
$$

and

$$
\left\|W_{2}\right\|_{2} \leq\left\|W_{0} e_{1}-e_{1}\right\|_{1}=2|\lambda| \varphi\left(\frac{1}{2|\lambda|}, n^{\prime}-1\right) .
$$

3. if $(\Lambda, \Omega)$ is of R4: $(-\Omega \Lambda)^{i^{\prime}-j^{\prime}}=\operatorname{diag}(R, \bar{R})$ and $R$ is of the form (A.3). Thus,

$$
\left\|W_{1}\right\|_{2} \leq \max \left\{\left\|W_{0} e_{1}\right\|_{1},\left\|\overline{W_{0}} e_{1}\right\|_{1}\right\}=2|\lambda| \varphi\left(\frac{1}{2|\lambda|}, n^{\prime}-1\right)+1,
$$

and

$$
\left\|W_{2}\right\|_{2} \leq \max \left\{\left\|W_{0} e_{1}-e_{1}\right\|_{1},\left\|\overline{W_{0}} e_{1}-e_{1}\right\|_{1}\right\}=2|\lambda| \varphi\left(\frac{1}{2|\lambda|}, n^{\prime}-1\right)
$$

\section{References}

[1] B. Allert and G. Müller. Brake-squeal simulation: An efficient approach to define countermeasures. In A. Cunha, E. Caetano, P. Ribeiro, and G. Müller, editors, Proceedings of the 9th International Conference on Structural Dynamics, EURODYN 2014, pages 3229-3235, July 2014.

[2] J. Barlow and J. Demmel. Computing accurate eigensystems of scaled diagonally dominant matrices. SIAM J. Numer. Anal., 27:762-791, 1990.

[3] H. Baumgartel. Analytic Perturbation Theory for Matrices and Operators. Birkhäuser, Basel, 1985.

[4] Timo Betcke, Nicholas J. Higham, Volker Mehrmann, Christian Schröder, and Françoise Tisseur. NLEVP: A collection of nonlinear eigenvalue problems. ACM Trans. Math. Softw., 39(2):7:1-7:28, February 2013.

[5] R. Bhatia. Matrix Analysis. Graduate Texts in Mathematics, vol. 169. Springer, New York, 1996.

[6] T. Damm and J. Homeyer. On indefinite damping and gyroscopic stabilization. In Proc. 18th IFAC World Congr., pages 7589-7593, August 2011.

[7] C. Davis and W. M. Kahan. The rotation of eigenvectors by a perturbation. III. SIAM J. Numer. Anal., 7:1-46, 1970.

[8] J. Demmel and W. Kahan. Accurate singular values of bidiagonal matrices. SIAM J. Sci. Statist. Comput., 11:873-912, 1990.

[9] J. Demmel and K. Veselić. Jacobi's method is more accurate than QR. SIAM J. Matrix Anal. Appl., 13(4):1204-1245, 1992.

[10] S. K. Godunov. Modern Aspects of Linear Algebra. Translations of Mathematical Monographs, vol. 175. AMS, Providence, RI, 1998.

[11] Israel Gohberg, Peter Lancaster, and Leiba Rodman. Matrix Polynomials. Academic Press, New York, 1982. 
[12] Israel Gohberg, Peter Lancaster, and Leiba Rodman. Indefinite Linear Algebra and Applications. Birkhäuser, Basel, Switzerland, 2005.

[13] L. Grubišić, N. Truhar, and S. Miodragović. The rotation of eigenspaces of perturbed matrix pairs II. Linear Multilinear Algebra, 62(8):1010-1031, 2014.

[14] L. Grubišić, N. Truhar, and K. Veselić. The rotation of eigenspaces of perturbed matrix pairs. Linear Algebra Appl., 462:4161-4178, 2012.

[15] Sayed-Mahdi Hashemi-Dehkordi, Musa Mailah, and Abd Rahim Abu Bakar. An active control method to reduce the effect of negative damping in disk brake system. In Innovative Technologies in Intelligent Systems and Industrial Applications, 2009. CITISIA 2009, pages 34-39. IEEE, 2009.

[16] W. Kahan. Accurate eigenvalues of a symmetric tridiagonal matrix. Technical Report CS41, Computer Science Department, Stanford University, Stanford, CA, 1966.

[17] T. Kato. Perturbation Theory for Linear Operators. Springer, New York, 1966.

[18] Wolfhard Kliem and Christian Pommer. Indefnite damping in mechanical systems and gyroscopic stabilization. Z. Angew. Math. Phys., 60(4):785-795, 2009.

[19] Peter Lancaster. Lambda-matrices and Vibrating Systems. Pergamon Press, 1966.

[20] Peter Lancaster and Leiba Rodman. Canonical forms for Hermitian matrix pairs under strict equivalence and congruence. SIAM Rev., 47(3):407-443, 2005.

[21] R.-C. Li. A bound on the solution to a structured Sylvester equation with an application to relative perturbation theory. SIAM J. Matrix Anal. Appl., 21(2):440-445, 1999.

[22] Ren-Cang Li. Relative perturbation theory: II. eigenspace and singular subspace variations. SIAM J. Matrix Anal. Appl., 20(2):471-492, 1999.

[23] Ren-Cang Li. On perturbations of matrix pencils with real spectra, a revisit. Math. Comp., 72:715728,2003 .

[24] Xin Liang and Ren-Cang Li. The hyperbolic quadratic eigenvalue problem. Forum of Mathematics, Sigma, 3(e13), 2015. 93 pages, doi:10.1017/fms.2015.14.

[25] B. N. Parlett. The Symmetric Eigenvalue Problem. SIAM, Philadelphia, 1998.

[26] Florian Petit, Dominic Lakatos, Werner Friedl, and Alin Albu-Schäffer. Dynamic trajectory generation for serial elastic actuated robots. In 10th International IFAC Symposium on Robot Control, 2012.

[27] G. W. Stewart and Ji-Guang Sun. Matrix Perturbation Theory. Academic Press, Boston, 1990.

[28] Françoise Tisseur and Karl Meerbergen. The quadratic eigenvalue problem. SIAM Rev., 43(2):235$286,2001$.

[29] Ninoslav Truhar and Suzana Miodragović. Relative perturbation theory for definite matrix pairs and hyperbolic eigenvalue problem. Appl. Numer. Math., 98:106-121, 2015.

[30] K. Veselić. Damped Oscillations of Linear Systems. Springer, Heidelberg, 2011.

[31] Songbin Wei and Imin Kao. Vibration analysis of wire and frequency response in the modern wiresaw manufacturing process. Journal of Sound and Vibration, 231(5):1383-1395, 2000. 